\title{
QUANTUM CURRENT ALGEBRAS ASSOCIATED WITH RATIONAL $R$-MATRIX
}

\author{
SLAVEN KOŽIĆ
}

\begin{abstract}
We study quantum current algebra $\mathrm{A}(\bar{R})$ associated with the rational $R$ matrix of $\mathfrak{g l}_{N}$ and we give explicit formulae for the elements of its center at the critical level. Due to Etingof-Kazhdan's construction, the level $c$ vacuum module $\mathcal{V}_{c}(\bar{R})$ for the algebra $\mathrm{A}(\bar{R})$ possesses a quantum vertex algebra structure for any complex number $c$. We prove that any module for the quantum vertex algebra $\mathcal{V}_{c}(\bar{R})$ is naturally equipped with a structure of restricted $\mathrm{A}(\bar{R})$-module of level $c$ and vice versa.
\end{abstract}

\section{INTRODUCTION}

Let $\mathfrak{g}$ be a Lie algebra over $\mathbb{C}$ equipped with a symmetric invariant bilinear form and let $\widehat{\mathfrak{g}}=\mathfrak{g} \otimes \mathbb{C}\left[t, t^{-1}\right] \oplus \mathbb{C} C$ be the corresponding affine Lie algebra. For any complex number $c$ we associate with $\widehat{\mathfrak{g}}$ the induced module

$$
V_{c}(\mathfrak{g})=U(\widehat{\mathfrak{g}}) \otimes_{U\left(\widehat{\mathfrak{g}}_{(\leqslant 0)}\right)} \mathbb{C}_{c}, \quad \text { where } \quad \widehat{\mathfrak{g}}_{(\leqslant 0)}=\coprod_{n \leqslant 0}\left(\mathfrak{g} \otimes t^{-n}\right) \oplus \mathbb{C} C
$$

and $\mathbb{C}_{c}=\mathbb{C}$ is an $U\left(\widehat{\mathfrak{g}}_{(\leqslant 0)}\right)$-module; the central element $C$ acts on $\mathbb{C}_{c}$ as scalar multiplication by $c$ and $\mathfrak{g} \otimes t^{-n}$ with $n \leqslant 0$ act trivially. By the results of I. B. Frenkel and Y.-C. Zhu in [6] and B.-H. Lian in [22], the space $V_{c}(\mathfrak{g})$ possesses a vertex algebra structure. Furthermore, any restricted $\widehat{\mathfrak{g}}$-module of level $c$ is naturally a module for the vertex algebra $V_{c}(\mathfrak{g})$ and vice versa; see [17, Chapter 6] for more details and references. In this paper, we study a certain quantum version of that result for $\mathfrak{g}=\mathfrak{g l}_{N}$.

The notion of quantum vertex operator algebra was introduced by P. Etingof and D. Kazhdan in [4]. They constructed examples of quantum vertex operator algebras by quantizing the quasiclassical structure on $V_{c}\left(\mathfrak{s l}_{N}\right)$ when the classical $r$-matrix on $\mathfrak{s l}_{N}$ is rational, trigonometric or elliptic. The corresponding vertex operator map was defined using quantum current $\mathcal{T}$, introduced by N. Yu. Reshetikhin and M. A. Semenov-TianShansky in [27], which satisfies a commutation relation of the form ${ }^{1}$

$$
\mathcal{T}_{1}(u) \bar{R}(u-v+h C)^{-1} \mathcal{T}_{2}(v) \bar{R}(u-v)=\bar{R}(-v+u)^{-1} \mathcal{T}_{2}(v) \bar{R}(-v+u-h C) \mathcal{T}_{1}(u) ;
$$

cf. also Ding's realization [2] of the quantum affine algebra in type $A$. Later on, the theory of quantum vertex algebras was further developed and generalized by H.-S. Li; see [18-21] and references therein. Specifically, in [21], an h-adic quantum vertex algebra was constructed on the level $c$ universal vacuum module for a certain cover of the double Yangian $\widehat{\mathrm{DY}\left(\mathfrak{s l}_{2}\right)}$ from [13] for any generic $c \in \mathbb{C}$. Moreover, it was proved that any highest

2010 Mathematics Subject Classification. 17B37 (Primary), 17B69 (Secondary).

Key words and phrases. Quantum current, Quantum vertex algebra, Double Yangian.

${ }^{1}$ We explain the precise meaning of (1) in Section 2.2. 
weight $\widehat{\mathrm{DY}\left(\mathfrak{s l}_{2}\right)}$-module of level $c$ is naturally equipped with a module structure for that $h$-adic quantum vertex algebra at the level $c$.

In this paper, we employ commutation relation (1), where $\bar{R}$ denotes the normalized Yang $R$-matrix, $h$ is a formal parameter and $C$ a central element, to define an associative algebra $\mathrm{A}(\bar{R})$ over the ring $\mathbb{C}[[h]]$, which we refer to as the quantum current algebra associated to $\bar{R}$. It is worth noting that the classical limit of (1) coincides with the commutation relation for the affine Lie algebra $\widehat{\mathfrak{g l}}_{N}$. We investigate properties of the algebra $\mathrm{A}(\bar{R})$ and, in particular, we use the fusion procedure originated in [11] to give explicit formulae for the elements of its center at the critical level.

Next, we introduce the notion of restricted $\mathrm{A}(\bar{R})$-module in parallel with the representation theory of the affine Lie algebras; see, e.g., [17, Chapter 6]. For any complex number $c$ we consider the vacuum module $\mathcal{V}_{c}(\bar{R})$ of level $c$ for the algebra $\mathrm{A}(\bar{R})$, which presents an example of restricted $\mathrm{A}(\bar{R})$-module. We show that, as a $\mathbb{C}[[h]]$-module, $\mathcal{V}_{c}(\bar{R})$ is isomorphic to the $h$-adically completed vacuum module $\mathcal{V}_{c}\left(\mathfrak{g l}_{N}\right)$ over the double Yangian for the Lie algebra $\mathfrak{g l}_{N}$. Hence, due to the aforementioned Etingof-Kazhdan's construction [4], the $\mathbb{C}[[h]]$-module $\mathcal{V}_{c}(\bar{R})=\mathcal{V}_{c}\left(\mathfrak{g l}_{N}\right)$ possesses a quantum vertex algebra structure. This structure was recently studied by N. Jing, A. Molev, F. Yang and the author in [9], where the center of $\mathcal{V}_{c}\left(\mathfrak{g l}_{N}\right)$ was determined, and also in [14], where a certain connection between quasi $\mathcal{V}_{c}\left(\mathfrak{g l}_{N}\right)$-modules and a class of reflection algebras of A. Molev and E. Ragoucy [25] was established.

The main result of this paper, Theorem 3.9 states that any module for the quantum vertex algebra $\mathcal{V}_{c}(\bar{R})$ is naturally equipped with a structure of restricted $\mathrm{A}(\bar{R})$-module of level $c$ and, conversely, that any restricted $\mathrm{A}(\bar{R})$-module of level $c$ is naturally equipped with a structure of module for the quantum vertex algebra $\mathcal{V}_{c}(\bar{R})$. Roughly speaking, the proof of the theorem relies on the fact that commutation relation (1) possesses a form similar to the $\mathcal{S}$-locality property, which is one of the fundamental quantum vertex algebra axioms.

We should mention that, starting with the work of E. K. Sklyanin [28], various classes of reflection algebras, which are defined via relations of the form similar to or same as

$$
R(u-v) B_{1}(u) R(u+v) B_{2}(v)=B_{2}(v) R(u+v) B_{1}(u) R(u-v),
$$

thus resembling commutation relation (1), were extensively studied. For more details the reader may consult $[7,10,15,16,23,25]$ and references therein. However, in contrast with (1), reflection equation (2) does not seem to directly give rise to the $\mathcal{S}$-locality property, i.e. to the quantum vertex algebra structure; see [14].

\section{Preliminaries}

In this section, we first recall some properties of the rational $R$-matrix. Next, we define the (completed) double Yangian for the Lie algebra $\mathfrak{g l}_{N}$ and its vacuum module. Finally, we recall the notions of quantum vertex algebra and module for a quantum vertex algebra, which play a central role in this paper. 
1.1. Rational $R$-matrix. Let $N \geqslant 2$ be an integer and let $h$ be a formal parameter. We follow [9, Section 2.2] to recall the definition and some basic properties of the rational $R$-matrix over the ring $\mathbb{C}[[h]]$. Consider the Yang R-matrix over $\mathbb{C}[[h]]$,

$$
R(u)=1-h P u^{-1} \in \operatorname{End} \mathbb{C}^{N} \otimes \text { End } \mathbb{C}^{N}\left[h, u^{-1}\right],
$$

where 1: $x \otimes y \mapsto x \otimes y$ is the identity and $P: x \otimes y \mapsto y \otimes x$ is the permutation operator on $\mathbb{C}^{N} \otimes \mathbb{C}^{N}$. There exists a unique series $g(u)$ in $1+u^{-1} \mathbb{C}\left[\left[u^{-1}\right]\right]$ such that

$$
g(u+N)=g(u)\left(1-u^{-2}\right) .
$$

The $R$-matrix $\bar{R}(u)=\bar{R}_{12}(u)=g(u / h) R(u)$ possesses the unitarity property

$$
\bar{R}_{12}(u) \bar{R}_{12}(-u)=1 \text {. }
$$

This as well as Yang R-matrix (1.3), the Yang-Baxter equation

$$
\bar{R}_{12}(u) \bar{R}_{13}(u+v) \bar{R}_{23}(v)=\bar{R}_{23}(v) \bar{R}_{13}(u+v) \bar{R}_{12}(u) .
$$

Both sides of (1.6) are operators on the triple tensor product $\left(\mathbb{C}^{N}\right)^{\otimes 3}$ and the subscripts indicate the copies of $\mathbb{C}^{N}$ on which the $R$-matrices are applied, e.g., $\bar{R}_{12}(u)=\bar{R}(u) \otimes 1$.

Due to (1.4), the $R$-matrix $\bar{R}(u)$ possesses the crossing symmetry properties,

$$
\left(\bar{R}_{12}(u)^{-1}\right)^{t_{1}} \bar{R}_{12}(u+h N)^{t_{1}}=1 \quad \text { and } \quad\left(\bar{R}_{12}(u)^{-1}\right)^{t_{2}} \bar{R}_{12}(u+h N)^{t_{2}}=1,
$$

where $t_{i}$ denotes the transposition applied on the tensor factor $i=1,2$. As in [9, Section 4.2 ], we can write (1.7) using the ordered product notation as

$$
\bar{R}_{12}(u)_{\mathrm{RL}}^{-1} \cdot \bar{R}_{12}(u+h N)=1 \quad \text { and } \quad \bar{R}_{12}(u)_{\mathrm{LR}}^{-1} \cdot \bar{R}_{12}(u+h N)=1,
$$

where the subscript RL (LR) in (1.8) indicates that the first tensor factor of $\bar{R}_{12}(u)^{-1}$ is applied from the right (left) while the second tensor factor of $\bar{R}_{12}(u)^{-1}$ is applied from the left (right). ${ }^{2}$ Indeed, (1.8) is obtained by applying the transposition $t_{1}$ on the first and $t_{2}$ on the second equality in (1.7).

1.2. Double Yangian for $\mathfrak{g l}_{N}$. The double Yangian $\mathrm{DY}\left(\mathfrak{g l}_{N}\right)$ for the Lie algebra $\mathfrak{g l}_{N}$ is the associative algebra over the ring $\mathbb{C}[[h]]$ generated by the central element $C$ and the elements $t_{i j}^{( \pm r)}$, where $i, j=1, \ldots, N$ and $r=1,2, \ldots$, subject to the defining relations

$$
\begin{aligned}
R(u-v) T_{1}(u) T_{2}(v) & =T_{2}(v) T_{1}(u) R(u-v), \\
R(u-v) T_{1}^{+}(u) T_{2}^{+}(v) & =T_{2}^{+}(v) T_{1}^{+}(u) R(u-v), \\
\bar{R}(u-v+h C / 2) T_{1}(u) T_{2}^{+}(v) & =T_{2}^{+}(v) T_{1}(u) \bar{R}(u-v-h C / 2),
\end{aligned}
$$

see $[3,4,8,14]$. The elements $T(u), T^{+}(u) \in \operatorname{End} \mathbb{C}^{N} \otimes \mathrm{DY}\left(\mathfrak{g l}_{N}\right)\left[\left[u^{\mp 1}\right]\right]$ are defined by

$$
T(u)=\sum_{i, j=1}^{N} e_{i j} \otimes t_{i j}(u) \quad \text { and } \quad T^{+}(u)=\sum_{i, j=1}^{N} e_{i j} \otimes t_{i j}^{+}(u),
$$

\footnotetext{
${ }^{2}$ Strictly speaking, notation used in [9] slightly differs. The equalities in (1.8) are expressed therein as ${ }^{r l} \bar{R}_{12}(u)^{-1} \bar{R}_{12}(u+h N)=1$ and ${ }^{l r} \bar{R}_{12}(u)^{-1} \bar{R}_{12}(u+h N)=1$.
} 
where the $e_{i j}$ denote the matrix units and the series $t_{i j}(u)$ and $t_{i j}^{+}(u)$ are defined by

$$
t_{i j}(u)=\delta_{i j}+h \sum_{r=1}^{\infty} t_{i j}^{(r)} u^{-r} \quad \text { and } \quad t_{i j}^{+}(u)=\delta_{i j}-h \sum_{r=1}^{\infty} t_{i j}^{(-r)} u^{r-1} .
$$

We indicate a copy of the matrix in the tensor product algebra $\left(\text { End } \mathbb{C}^{N}\right)^{\otimes m} \otimes \operatorname{DY}\left(\mathfrak{g l}_{N}\right)$ by subscripts, so that, for example, we have

$$
T_{k}(u)=\sum_{i, j=1}^{N} 1^{\otimes(k-1)} \otimes e_{i j} \otimes 1^{\otimes(m-k)} \otimes t_{i j}(u) .
$$

In particular, we have $m=2$ and $k=1,2$ in defining relations (1.9)-(1.11).

The Yangian $\mathrm{Y}\left(\mathfrak{g l}_{N}\right)$ is the subalgebra of $\mathrm{DY}\left(\mathfrak{g l}_{N}\right)$ generated by the elements $t_{i j}^{(r)}$, where $i, j=1, \ldots, N$ and $r=1,2, \ldots$ The dual Yangian $\mathrm{Y}^{+}\left(\mathfrak{g l}_{N}\right)$ is the subalgebra of the double Yangian $\operatorname{DY}\left(\mathfrak{g l}_{N}\right)$ generated by the elements $t_{i j}^{(-r)}$, where $i, j=1, \ldots, N$ and $r=1,2, \ldots$ For any $c \in \mathbb{C}$ denote by $\mathrm{DY}_{c}\left(\mathfrak{g l}_{N}\right)$ the double Yangian at the level $c$, which is defined as the quotient of the algebra $\mathrm{DY}\left(\mathfrak{g l}_{N}\right)$ by the ideal generated by the element $C-c$.

For any integer $p \geqslant 1$ let $\mathrm{I}_{p}\left(\mathfrak{g l}_{N}\right)$ be the left ideal in $\mathrm{DY}_{c}\left(\mathfrak{g l}_{N}\right)$ generated by all elements $t_{i j}^{(r)}$, where $i, j=1, \ldots, N$ and $r \geqslant p$. Introduce the completion of the double Yangian $\mathrm{DY}_{c}\left(\mathfrak{g l}_{N}\right)$ at the level $c$ as the inverse limit

$$
\widetilde{\mathrm{DY}}_{c}\left(\mathfrak{g l}_{N}\right)=\lim _{\longleftarrow} \mathrm{DY}_{c}\left(\mathfrak{g l}_{N}\right) / \mathrm{I}_{p}\left(\mathfrak{g l}_{N}\right) .
$$

1.3. Vacuum module over the double Yangian. Let $V$ be an arbitrary $\mathbb{C}[[h]]$ module. The $h$-adic topology on $V$ is the topology generated by the basis $v+h^{n} V$, where $v \in V$ and $n \in \mathbb{Z}_{\geqslant 1}$. Recall that $V$ is said to be torsion-free if $h v \neq 0$ for all nonzero $v \in V$ and that $V$ is said to be separable if $\cap_{m \geqslant 1} h^{m} V=0$. A $\mathbb{C}[[h]]$-module $\mathrm{V}$ is said to be topologically free if it is separable, torsion-free and complete with respect to the $h$-adic topology. For more details on topologically free $\mathbb{C}[[h]]$-modules see $[12$, Chapter XVI].

We now introduce the vacuum module over the double Yangian as in [9, Section 4.2]. Let $\mathrm{W}_{c}\left(\mathfrak{g l}_{N}\right)$ be the left ideal in $\mathrm{DY}_{c}\left(\mathfrak{g l}_{N}\right)$ generated by the elements $t_{i j}^{(r)}$, where $i, j=1, \ldots, N$ and $r=1,2, \ldots$ By the Poincaré-Birkhoff-Witt theorem for the double Yangian, see [9, Theorem 2.2], the quotient

$$
\mathrm{DY}_{c}\left(\mathfrak{g l}_{N}\right) / \mathrm{W}_{c}\left(\mathfrak{g l}_{N}\right)
$$

is isomorphic, as a $\mathbb{C}[[h]]$-module, to the dual Yangian $\mathrm{Y}^{+}\left(\mathfrak{g l}_{N}\right)$. The vacuum module $\mathcal{V}_{c}\left(\mathfrak{g l}_{N}\right)$ at the level $c$ over the double Yangian is defined as the $h$-adic completion of quotient (1.13). The vacuum module $\mathcal{V}_{c}\left(\mathfrak{g l}_{N}\right)$ is topologically free $\widetilde{\mathrm{DY}}_{c}\left(\mathfrak{g l}_{N}\right)$-module. We denote by 1 the image of the unit $1 \in \mathrm{DY}_{c}\left(\mathfrak{g l}_{N}\right)$ in quotient (1.13).

For positive integers $n$ and $m$ introduce functions depending on the variable $z$ and the families of variables $u=\left(u_{1}, \ldots, u_{n}\right)$ and $v=\left(v_{1}, \ldots, v_{m}\right)$ with values in the space $\left(\text { End } \mathbb{C}^{N}\right)^{\otimes n} \otimes\left(\text { End } \mathbb{C}^{N}\right)^{\otimes m}$ by

$$
\begin{aligned}
& \bar{R}_{n m}^{12}(u|v| z)=\prod_{i=1, \ldots, n j=n+1, \ldots, n+m} \prod_{i j} \bar{R}_{i j}\left(z+u_{i}-v_{j-n}\right), \\
& \overleftarrow{\leftarrow}_{n m}^{12}(u|v| z)=\prod_{i=1, \ldots, n} \prod_{j=n+1, \ldots, n+m} \bar{R}_{i j}\left(z+u_{i}-v_{j-n}\right)
\end{aligned}
$$


where the arrows indicate the order of the factors. For example, we have

$$
\bar{R}_{22}^{12}(u|v| z)=\bar{R}_{14} \bar{R}_{13} \bar{R}_{24} \bar{R}_{23} \quad \text { and } \quad \bar{R}_{22}^{12}(u|v| z)=\bar{R}_{23} \bar{R}_{24} \bar{R}_{13} \bar{R}_{14},
$$

where $\bar{R}_{i j}=\bar{R}_{i j}\left(z+u_{i}-v_{j-n}\right)$.

We adopt the following expansion convention. For any variables $x_{1}, \ldots, x_{k}$ expressions of the form $\left(x_{1}+\ldots+x_{k}\right)^{s}$ with $s<0$ should be expanded in nonnegative powers of the variables $x_{2}, \ldots, x_{k}$. In particular, expressions of the form $\left(z+u_{i}-v_{j-n}\right)^{s}$ with $s<0$ should be expanded in negative powers of the variable $z$, so that (1.14) and (1.15) contain only nonnegative powers of the variables $u_{1}, \ldots, u_{n}$ and $v_{1}, \ldots, v_{m}$. Also, we write

$$
\bar{R}_{n m}^{12}(u \mid v)=\bar{R}_{n m}^{12}(u|v| 0) \text { and } \overleftarrow{\leftarrow}_{n m}^{12}(u \mid v)=\overleftarrow{\leftarrow}_{n m}^{12}(u|v| 0),
$$

where, due to the aforementioned expansion convention, expressions of the form $\left(u_{i}-\right.$ $\left.v_{j-n}\right)^{s}$ with $s<0$, which appear in (1.16), are expanded in negative powers of the variable $u_{i}$, so that they contain only nonnegative powers of $v_{j-n}$. The functions $R_{n m}^{12}(u|v| z)$ and $\overleftarrow{R}_{n m}^{12}(u|v| z)$ corresponding to Yang $R$-matrix (1.3) can be defined analogously.

Introduce the operators on $\left(\text { End } \mathbb{C}^{N}\right)^{\otimes n} \otimes \mathcal{V}_{c}\left(\mathfrak{g l}_{N}\right)$ by

$$
T_{[n]}^{+}(u \mid z)=T_{1}^{+}\left(z+u_{1}\right) \ldots T_{n}^{+}\left(z+u_{n}\right) \quad \text { and } \quad T_{[n]}(u \mid z)=T_{1}\left(z+u_{1}\right) \ldots T_{n}\left(z+u_{n}\right) .
$$

Note that, due to our expansion convention, the operator $T_{[n]}(u \mid z)$ contains only nonnegative powers of the variables $u_{1}, \ldots, u_{n}$. Also, we write

$$
T_{[n]}^{+}(u)=T_{1}^{+}\left(u_{1}\right) \ldots T_{n}^{+}\left(u_{n}\right) \quad \text { and } \quad T_{[n]}(u)=T_{1}\left(u_{1}\right) \ldots T_{n}\left(u_{n}\right) .
$$

Note that both expressions in (1.17) can be viewed as series with coefficients in the double Yangian. Using defining relations (1.9)-(1.11) one can verify the following equations for the operators on

$$
\underbrace{\left(\text { End } \mathbb{C}^{N}\right)^{\otimes n}}_{1} \otimes \underbrace{\left(\operatorname{End} \mathbb{C}^{N}\right)^{\otimes m}}_{2} \otimes \underbrace{\mathcal{V}_{c}\left(\mathfrak{g l}_{N}\right)}_{3}
$$

which were given in [4]:

$$
\begin{aligned}
& R_{n m}^{12}\left(u|v| z_{1}-z_{2}\right) T_{[n]}^{+13}\left(u \mid z_{1}\right) T_{[m]}^{+23}\left(v \mid z_{2}\right)=T_{[m]}^{+23}\left(v \mid z_{2}\right) T_{[n]}^{+13}\left(u \mid z_{1}\right) R_{n m}^{12}\left(u|v| z_{1}-z_{2}\right) \\
& R_{n m}^{12}\left(u|v| z_{1}-z_{2}\right) T_{[n]}^{13}\left(u \mid z_{1}\right) T_{[m]}^{23}\left(v \mid z_{2}\right)=T_{[m]}^{23}\left(v \mid z_{2}\right) T_{[n]}^{13}\left(u \mid z_{1}\right) R_{n m}^{12}\left(u|v| z_{1}-z_{2}\right) \\
& \bar{R}_{n m}^{12}\left(u|v| z_{1}-z_{2}+h c / 2\right) T_{[n]}^{13}\left(u \mid z_{1}\right) T_{[m]}^{+23}\left(v \mid z_{2}\right) \\
& =T_{[m]}^{+23}\left(v \mid z_{2}\right) T_{[n]}^{13}\left(u \mid z_{1}\right) \bar{R}_{n m}^{12}\left(u|v| z_{1}-z_{2}-h c / 2\right)
\end{aligned}
$$

In relations (1.19)-(1.21), we use superscripts to indicate tensor factors in accordance with (1.18). For example, $T_{[n]}^{+13}\left(u \mid z_{1}\right)$ is applied on tensor factors $1, \ldots, n$ and $n+m+1$, and $T_{[m]}^{+23}\left(v \mid z_{2}\right)$ is applied on tensor factors $n+1, \ldots, n+m$ and $n+m+1$. We will often use such notation throughout this paper.

1.4. Quantum vertex algebras. The definitions in this section are presented in the form which we find to be suitable for the setting of this paper. In particular, the next definition of quantum vertex algebra coincides with [9, Definition 3.1]. It presents a minor modification of the original definition of quantum vertex operator algebra given by Etingof and Kazhdan in [4], as explained in [9, Remark 3.2 and 3.4]. For more details on the 
axiomatics of quantum vertex algebras and related structures the reader may consult $[4,21]$. From now on, the tensor products are understood as $h$-adically completed.

Definition 1.1. A quantum vertex algebra is a quintuple $(V, Y, \mathbf{1}, D, \mathcal{S})$ which satisfies the following axioms:

(1) $V$ is a topologically free $\mathbb{C}[[h]]$-module.

(2) $Y$ is a $\mathbb{C}[[h]]$-module map (the vertex operator map)

$$
\begin{aligned}
Y: V \otimes V & \rightarrow V((z))[[h]] \\
u \otimes v & \mapsto Y(z)(u \otimes v)=Y(u, z) v=\sum_{r \in \mathbb{Z}} u_{r} v z^{-r-1}
\end{aligned}
$$

which satisfies the weak associativity: for any $u, v, w \in V$ and $n \in \mathbb{Z}_{\geqslant 0}$ there exists $r \in \mathbb{Z}_{\geqslant 0}$ such that

$$
\left(z_{0}+z_{2}\right)^{r} Y\left(u, z_{0}+z_{2}\right) Y\left(v, z_{2}\right) w-\left(z_{0}+z_{2}\right)^{r} Y\left(Y\left(u, z_{0}\right) v, z_{2}\right) w \in h^{n} V\left[\left[z_{0}^{ \pm 1}, z_{2}^{ \pm 1}\right]\right] .
$$

(3) $\mathbf{1}$ is an element of $V$ (the vacuum vector) which satisfies

$$
Y(\mathbf{1}, z) v=v \text { for all } v \in V,
$$

and for any $v \in V$ the series $Y(v, z) \mathbf{1}$ is a Taylor series in $z$ with the property

$$
\lim _{z \rightarrow 0} Y(v, z) \mathbf{1}=v .
$$

(4) $D$ is a $\mathbb{C}[[h]]$-module map $V \rightarrow V$ which satisfies

$$
D \mathbf{1}=0 \quad \text { and } \quad \frac{d}{d z} Y(v, z)=[D, Y(v, z)] \quad \text { for all } v \in V .
$$

(5) $\mathcal{S}=\mathcal{S}(z)$ is a $\mathbb{C}[[h]]$-module map $V \otimes V \rightarrow V \otimes V \otimes \mathbb{C}((z))$ which satisfies the shift condition

$$
[D \otimes 1, \mathcal{S}(z)]=-\frac{d}{d z} \mathcal{S}(z)
$$

the Yang-Baxter equation

$$
\mathcal{S}_{12}\left(z_{1}\right) \mathcal{S}_{13}\left(z_{1}+z_{2}\right) \mathcal{S}_{23}\left(z_{2}\right)=\mathcal{S}_{23}\left(z_{2}\right) \mathcal{S}_{13}\left(z_{1}+z_{2}\right) \mathcal{S}_{12}\left(z_{1}\right),
$$

the unitarity condition

$$
\mathcal{S}_{21}(z)=\mathcal{S}^{-1}(-z),
$$

and the $\mathcal{S}$-locality: for any $u, v \in V$ and $n \in \mathbb{Z}_{\geqslant 0}$ there exists $r \in \mathbb{Z}_{\geqslant 0}$ such that

$$
\begin{aligned}
& \left(z_{1}-z_{2}\right)^{r} Y\left(z_{1}\right)\left(1 \otimes Y\left(z_{2}\right)\right)\left(\mathcal{S}\left(z_{1}-z_{2}\right)(u \otimes v) \otimes w\right) \\
& \quad-\left(z_{1}-z_{2}\right)^{r} Y\left(z_{2}\right)\left(1 \otimes Y\left(z_{1}\right)\right)(v \otimes u \otimes w) \in h^{n} V\left[\left[z_{1}^{ \pm 1}, z_{2}^{ \pm 1}\right]\right] \quad \text { for all } w \in V .
\end{aligned}
$$

It was proved in [21] that the $\mathcal{S}$-Jacobi identity

$$
\begin{aligned}
& z_{0}^{-1} \delta\left(\frac{z_{1}-z_{2}}{z_{0}}\right) Y\left(z_{1}\right)\left(1 \otimes Y\left(z_{2}\right)\right)(u \otimes v \otimes w) \\
& -z_{0}^{-1} \delta\left(\frac{z_{2}-z_{1}}{-z_{0}}\right) Y\left(z_{2}\right)\left(1 \otimes Y\left(z_{1}\right)\right)\left(\mathcal{S}\left(-z_{0}\right)(v \otimes u) \otimes w\right) \\
& \quad=z_{2}^{-1} \delta\left(\frac{z_{1}-z_{0}}{z_{2}}\right) Y\left(Y\left(u, z_{0}\right) v, z_{2}\right) w \quad \text { for all } u, v, w \in V
\end{aligned}
$$


is equivalent to weak associativity (1.22) and $\mathcal{S}$-locality (1.29). In what follows, the (appropriately modified) $\mathcal{S}$-Jacobi identity is used to define the notion of module for a quantum vertex algebra. Originally, modules for $h$-adic nonlocal vertex algebras, which present a generalization of quantum vertex algebras, as well as modules for some related structures, were introduced and studied by Li; see, e.g., [18-21] and references therein.

Definition 1.2. Let $(V, Y, 1, D, \mathcal{S})$ be a quantum vertex algebra. A $V$-module is a pair $\left(W, Y_{W}\right)$, where $W$ is a topologically free $\mathbb{C}[[h]]$-module and

$$
\begin{aligned}
Y_{W}(z): V \otimes W & \rightarrow W((z))[[h]] \\
v \otimes w & \mapsto Y_{W}(z)(v \otimes w)=Y_{W}(v, z) w=\sum_{r \in \mathbb{Z}} v_{r} w z^{-r-1}
\end{aligned}
$$

is a $\mathbb{C}[[h]]$-module map which satisfies the $\mathcal{S}$-Jacobi identity

$$
\begin{aligned}
& z_{0}^{-1} \delta\left(\frac{z_{1}-z_{2}}{z_{0}}\right) Y_{W}\left(z_{1}\right)\left(1 \otimes Y_{W}\left(z_{2}\right)\right)(u \otimes v \otimes w) \\
& -z_{0}^{-1} \delta\left(\frac{z_{2}-z_{1}}{-z_{0}}\right) Y_{W}\left(z_{2}\right)\left(1 \otimes Y_{W}\left(z_{1}\right)\right)\left(\mathcal{S}\left(-z_{0}\right)(v \otimes u) \otimes w\right) \\
& \quad=z_{2}^{-1} \delta\left(\frac{z_{1}-z_{0}}{z_{2}}\right) Y_{W}\left(Y\left(u, z_{0}\right) v, z_{2}\right) w \quad \text { for all } u, v \in V \text { and } w \in W
\end{aligned}
$$

and

$$
Y_{W}(\mathbf{1}, z) w=w \quad \text { for all } w \in W .
$$

Let $W_{1}$ be a topologically free $\mathbb{C}[[h]]$-submodule of $W$. A pair $\left(W_{1}, Y_{W_{1}}\right)$ is said to be a $V$-submodule of $W$ if $Y_{W}(v, z) w_{1}$ belongs to $W_{1}$ for all $v \in V$ and $w_{1} \in W_{1}$, where $Y_{W_{1}}$ denotes the restriction and corestriction of $Y_{W}$,

$$
Y_{W_{1}}(z)=\left.Y_{W}(z)\right|_{V \otimes W_{1}} ^{W_{1}}: V \otimes W_{1} \rightarrow W_{1}((z))[[h]] .
$$

The next lemma, which we use in the proof of Theorem 3.9, can be viewed as an $h$ adic analogue of [20, Remark 2.5]; see also [17, Theorem 4.4.5]. Roughly speaking, it is a consequence of [18, Lemma 2.1] and the fact that for any quantum vertex algebra $V$ and $V$-module $W$ the quotient $V / h^{n} V$ is a weak quantum vertex algebra over $\mathbb{C}$ and $W / h^{n} W$ is a $V / h^{n} V$-module for all $n \geqslant 1$; see [21] for details.

Lemma 1.3. Let $(V, Y, \mathbf{1}, D, \mathcal{S})$ be a quantum vertex algebra and let $W$ be a topologically free $\mathbb{C}[[h]]$-module equipped with $\mathbb{C}[[h]]$-module map

$$
\begin{aligned}
Y_{W}(z): V \otimes W & \rightarrow W((z))[[h]] \\
v \otimes w & \mapsto Y_{W}(z)(v \otimes w)=Y_{W}(v, z) w=\sum_{r \in \mathbb{Z}} v_{r} w z^{-r-1}
\end{aligned}
$$

which satisfies $Y_{W}(\mathbf{1}, z) w=w$ for all $w \in W$ and the weak associativity: for any $u, v \in V$, $w \in W$ and $n \in \mathbb{Z}_{\geqslant 0}$ there exists $r \in \mathbb{Z}_{\geqslant 0}$ such that

$$
\begin{aligned}
& \left(z_{0}+z_{2}\right)^{r} Y_{W}\left(u, z_{0}+z_{2}\right) Y_{W}\left(v, z_{2}\right) w \\
& \quad-\left(z_{0}+z_{2}\right)^{r} Y_{W}\left(Y\left(u, z_{0}\right) v, z_{2}\right) w \in h^{n} W\left[\left[z_{0}^{ \pm 1}, z_{2}^{ \pm 1}\right]\right] .
\end{aligned}
$$


Then $\left(W, Y_{W}\right)$ is a $V$-module. In particular, the $\mathcal{S}$-locality holds, i.e. for any $u, v \in V$ and $n \in \mathbb{Z}_{\geqslant 0}$ there exists $r \in \mathbb{Z}_{\geqslant 0}$ such that

$$
\begin{aligned}
& \left(z_{1}-z_{2}\right)^{r} Y_{W}\left(z_{1}\right)\left(1 \otimes Y_{W}\left(z_{2}\right)\right)\left(\mathcal{S}\left(z_{1}-z_{2}\right)(u \otimes v) \otimes w\right) \\
& -\left(z_{1}-z_{2}\right)^{r} Y_{W}\left(z_{2}\right)\left(1 \otimes Y_{W}\left(z_{1}\right)\right)(v \otimes u \otimes w) \in h^{n} W\left[\left[z_{1}^{ \pm 1}, z_{2}^{ \pm 1}\right]\right] \quad \text { for all } w \in W .
\end{aligned}
$$

Proof. The lemma can be proved by arguing as in [21, Remark 2.16] and using [20, Remark 2.5] and [21, Proposition 2.24].

\section{Quantum CURREnt algebras}

In this section, we introduce quantum current algebra and study its properties. In particular, we construct an action of the quantum current algebra on the vacuum module over the double Yangian, which is an important ingredient of the proof of our main result, Theorem 3.9 in Section 3. In the end, we give explicit formulae for families of central elements of the quantum current algebra at the critical level.

2.1. Preliminaries. Our first goal is to introduce the quantum current algebra $\mathrm{A}(\bar{R})$. However, its defining relations, i.e. the coefficients in commutation relation (2.8) with respect to the variables $u$ and $v$, are given in terms of certain infinite sums. In order to handle such expressions and employ commutation relation (2.8), we introduce the appropriate completion of the corresponding free algebra.

For any integer $N \geqslant 2$ let $\mathrm{F}(N)$ be the associative algebra over the ring $\mathbb{C}[[h]]$ generated by the elements $1, C$ and $\tau_{i j}^{(r)}$, where $i, j=1, \ldots, N$ and $r \in \mathbb{Z}$, subject to the following defining relations:

$$
C \cdot x=x \cdot C \quad \text { and } \quad 1 \cdot x=x \cdot 1=x \quad \text { for all } x \in \mathrm{F}(N) .
$$

Hence 1 is the unit and $C$ is a central element in $\mathrm{F}(N)$. Arrange all $\tau_{i j}^{(r)}$ into Laurent series

$$
\tau_{i j}(u)=\delta_{i j}-h \sum_{r \in \mathbb{Z}} \tau_{i j}^{(r)} u^{-r-1} \in \mathrm{F}(N)\left[\left[u^{ \pm 1}\right]\right], \quad \text { where } i, j=1, \ldots, N,
$$

and introduce the elements $\mathcal{T}(u)$ in End $\mathbb{C}^{N} \otimes \mathrm{F}(N)\left[\left[u^{ \pm 1}\right]\right]$ by

$$
\mathcal{T}(u)=\sum_{i, j=1}^{N} e_{i j} \otimes \tau_{i j}(u),
$$

where the $e_{i j}$ denote the matrix units.

For any integer $n \geqslant 1$ let $\mathrm{I}_{n}(N)$ be the left ideal in the algebra $\mathrm{F}(N)$ generated by all elements $\tau_{i j}^{(r)}$, where $i, j=1, \ldots, N$ and $r \geqslant n-1$. Introduce the completion of the algebra $\mathrm{F}(N)$ as the inverse limit

$$
\mathrm{F}^{\prime}(N)=\lim _{\longleftarrow} \mathrm{F}(N) / \mathrm{I}_{n}(N)
$$

The algebra $\mathrm{F}^{\prime}(N)$ is naturally equipped with the $h$-adic topology. Let $\widetilde{\mathrm{F}}(N)$ be the $h$-adic completion of $\mathrm{F}^{\prime}(N)$, i.e. $\widetilde{\mathrm{F}}(N)=\mathrm{F}^{\prime}(N)[[h]]$. For any integer $n \geqslant 1$ denote by $\mathrm{I}_{n}^{h}(N)$ the $h$-adically completed left ideal in $\widetilde{\mathrm{F}}(N)$ generated by $\mathrm{I}_{n}(N)$ and $h^{n} \cdot 1$. Recall the subscript 
notation from Section 1.2 and the expansion convention from Section 1.3. Consider the following expressions:

$$
\begin{aligned}
& \overline{\mathcal{T}}_{[2]}(u, v)=\mathcal{T}_{1}(u) \bar{R}(u-v+h C)^{-1} \mathcal{T}_{2}(v) \bar{R}(u-v), \\
& \underline{\mathcal{T}}_{[2]}(u, v)=\bar{R}(-v+u)^{-1} \mathcal{T}_{2}(v) \bar{R}(-v+u-h C) \mathcal{T}_{1}(u) .
\end{aligned}
$$

Lemma 2.1. The expressions $\overline{\mathcal{T}}_{[2]}(u, v)$ and $\underline{\mathcal{T}}_{[2]}(u, v)$ are well-defined elements of

$$
\text { End } \mathbb{C}^{N} \otimes \text { End } \mathbb{C}^{N} \otimes \widetilde{\mathrm{F}}(N)\left[\left[u^{ \pm 1}, v^{ \pm 1}\right]\right] \text {. }
$$

Moreover, for any integer $n \geqslant 1$ the elements $\overline{\mathcal{T}}_{[2]}(u, v)$ and $\underline{\mathcal{T}}_{[2]}(v, u)^{3}$ modulo $\mathrm{I}_{n}^{h}(N)$ belong to

$$
\text { End } \mathbb{C}^{N} \otimes \text { End } \mathbb{C}^{N} \otimes \mathrm{F}(N)\left[\left[u^{ \pm 1}\right]\right]((v))
$$

Proof. The matrix entries of $\bar{R}(u-v+h C)^{-1}$ and $\bar{R}(u-v)$ belong to $\mathbb{C}\left[u^{-1}\right][[h C, h, v]]$. For any integer $n \geqslant 1$ the matrix entries of $\mathcal{T}(v)$ modulo $\mathrm{I}_{n}(N)$ belong to $\mathrm{F}(N)((v))$. Therefore, the expression $\bar{R}(u-v+h C)^{-1} \mathcal{T}_{2}(v) \bar{R}(u-v)$ is a well-defined element of $(2.3)$ and its matrix entries modulo $\mathrm{I}_{n}^{h}(N)$ belong to $\mathrm{F}(N)\left[u^{-1}\right]((v))$. Hence, by left multiplying this expression by $\mathcal{T}_{1}(u)$, we conclude that $\overline{\mathcal{T}}_{[2]}(u, v)$ is a well-defined element of (2.3) and, furthermore, that $\overline{\mathcal{T}}_{[2]}(u, v)$ modulo $\mathrm{I}_{n}^{h}(N)$ belongs to (2.4). The corresponding statements for $\underline{\mathcal{T}}_{[2]}(v, u)$ can be verified analogously.

By Lemma 2.1, there exist elements $\bar{\tau}_{i j k l}^{(r, s)}$ and $\underline{\tau}_{i j k l}^{(r, s)}$ in $\widetilde{\mathrm{F}}(N)$, where $i, j, k, l=1, \ldots, N$ and $r, s \in \mathbb{Z}$, such that

$$
\begin{aligned}
& \overline{\mathcal{T}}_{[2]}(u, v)=\sum_{i, j, k, l=1}^{N} \sum_{r, s \in \mathbb{Z}} e_{i j} \otimes e_{k l} \otimes \bar{\tau}_{i j k l}^{(r, s)} u^{-r-1} v^{-s-1}, \\
& \underline{\mathcal{T}}_{[2]}(u, v)=\sum_{i, j, k, l=1}^{N} \sum_{r, s \in \mathbb{Z}} e_{i j} \otimes e_{k l} \otimes \underline{\tau}_{i j k l}^{(r, s)} u^{-r-1} v^{-s-1} .
\end{aligned}
$$

2.2. Quantum current algebra. We are now prepared to introduce the quantum current algebra $\mathrm{A}(\bar{R})$. Let $\mathrm{J}(N)$ be the ideal in the algebra $\widetilde{\mathrm{F}}(N)$ generated by all elements $\bar{\tau}_{i j k l}^{(r, s)}-\underline{\tau}_{i j k l}^{(r, s)}$, where $i, j, k, l=1, \ldots, N$ and $r, s \in \mathbb{Z}$. Introduce its completion as the inverse limit

$$
\mathrm{J}^{\prime}(N)=\lim _{\longleftarrow} \mathrm{J}(N) / \mathrm{J}(N) \cap \mathrm{I}_{n}(N) .
$$

The $h$-adic completion $\widetilde{\left[\mathrm{J}^{\prime}(N)\right]}$ of

$$
\left[\mathrm{J}^{\prime}(N)\right]=\left\{a \in \widetilde{\mathrm{F}}(N): h^{n} a \in \mathrm{J}^{\prime}(N) \text { for some } n \geqslant 0\right\}
$$

is also an ideal in $\widetilde{\mathrm{F}}(N)$. Define the quantum current algebra $\mathrm{A}(\bar{R})$ as the quotient of the algebra $\widetilde{\mathrm{F}}(N)$ by the ideal $\left[\widetilde{\mathrm{J}^{\prime}(N)}\right]$,

$$
\mathrm{A}(\bar{R})=\widetilde{\mathrm{F}}(N) / \widetilde{\left[\mathrm{J}^{\prime}(N)\right]} .
$$

Clearly, the algebra $\mathrm{A}(\bar{R})$ is equipped with the $h$-adic topology.

Proposition 2.2. The algebra $\mathrm{A}(\bar{R})$ is topologically free.

\footnotetext{
${ }^{3}$ Notice the swapped variables in this term.
} 
Proof. We will prove that the algebra $\mathrm{A}(\bar{R})$ is torsion-free, separated and $h$-adically complete. Let $a$ be an arbitrary element in $\mathrm{A}(\bar{R})$. Choose any element $b$ in $\widetilde{\mathrm{F}}(N)$ such that its image, with respect to the canonical map $\pi: \widetilde{\mathrm{F}}(N) \rightarrow \widetilde{\mathrm{F}}(N) /\left[\widetilde{\left.\mathrm{J}^{\prime}(N)\right]}\right.$, equals $a$. By [21, Proposition 3.7] we have

$$
\left.\widetilde{\left[\mathrm{J}^{\prime}(N)\right]}=\left\{a \in \widetilde{\mathrm{F}}(N): h^{n} a \in \widetilde{\left[\mathrm{J}^{\prime}(N)\right.}\right] \text { for some } n \geqslant 0\right\} \text {. }
$$

Suppose that $h a=0$. Then $h b$ belongs to $\widetilde{\left[\mathrm{J}^{\prime}(N)\right]}$, so we conclude by $(2.7)$ that $b$ belongs to $\left[\widetilde{\mathrm{J}^{\prime}(N)}\right]$. This implies $a=\pi(b)=0$, so the algebra $\mathrm{A}(\bar{R})$ is torsion-free.

Suppose that $a$ belongs to $h^{n} \mathrm{~A}(\bar{R})$ for all $n \geqslant 1$. This implies

$$
b \in \widetilde{\left[\mathrm{J}^{\prime}(N)\right]} \bmod h^{n} \widetilde{\mathrm{F}}(N) \quad \text { for all } n \geqslant 1
$$

Hence we can construct a sequence $\left(b_{n}\right)_{n}$ in $\widetilde{\left[\mathrm{J}^{\prime}(N)\right]}$ such that $b=\lim _{n} b_{n}$, with respect to the $h$-adic topology. Since the ideal $\widetilde{\left[\mathrm{J}^{\prime}(N)\right]}$ is $h$-adically complete, we conclude that $\lim _{n} b_{n}=b$ belongs to $\left[\widetilde{\mathrm{J}^{\prime}(N)}\right]$, which implies $a=\pi(b)=0$. Therefore, $\cap_{n \geqslant 1} h^{n} \mathrm{~A}(\bar{R})=0$, so the algebra $\mathrm{A}(\bar{R})$ is separated.

It remains to prove that the algebra $\mathrm{A}(\bar{R})$ is $h$-adically complete. Let $\left(a_{n}\right)_{n}$ be a Cauchy sequence in $\mathrm{A}(\bar{R})$. There exists an increasing and unbounded sequence of nonnegative integers $\left(m_{n}\right)_{n}$ such that $a_{n+1}-a_{n}$ belongs to $h^{m_{n}} \mathrm{~A}(\bar{R})$ for all $n$. Let $\left(b_{n}\right)_{n}$ be any sequence in $\widetilde{\mathrm{F}}(N)$ such that $\pi\left(b_{n}\right)=a_{n}$ for all $n$. There exist elements $c_{n} \in \widetilde{\mathrm{F}}(N)$ and $\left.d_{n} \in \widetilde{\mathrm{J}^{\prime}(N)}\right]$ such that $b_{n+1}-b_{n}=h^{m_{n}} c_{n}+d_{n}$ for all $n$. Consider the sequence $\left(e_{n}\right)_{n}$ in $\widetilde{\mathrm{F}}(N)$ defined by $e_{n}=b_{n}-d_{n-1}-\ldots-d_{1}$. We have $e_{n+1}-e_{n}=h^{m_{n}} c_{n}$ for all $n$, so $\left(e_{n}\right)_{n}$ is a Cauchy sequence in $\widetilde{\mathrm{F}}(N)$. Since $\widetilde{\mathrm{F}}(N)$ is $h$-adically complete, there exists $e \in \widetilde{\mathrm{F}}(N)$ such that $\lim _{n} e_{n}=e$. Hence, for every $k \geqslant 0$ there exists $l \geqslant 1$ such that $e-e_{n} \in h^{k} \widetilde{\mathrm{F}}(N)$ for all $n \geqslant l$. Since $\pi\left(e_{n}\right)=\pi\left(b_{n}\right)=a_{n}$, this implies $\pi(e)-a_{n} \in h^{k} \mathrm{~A}(\bar{R})$ for all $n \geqslant l$. Therefore, the limit of the Cauchy sequence $\left(a_{n}\right)_{n}$ does exist in $\mathrm{A}(\bar{R})$ (and is equal to $\pi(e))$, so we conclude that the algebra $\mathrm{A}(\bar{R})$ is $h$-adically complete.

From now on, we denote the images of the elements $1, C$ and $\tau_{i j}^{(r)}$ in quotient (2.6) by 1, $C$ and $\tau_{i j}^{(r)}$ respectively. Also, we denote by $\tau_{i j}(u)$ and $\mathcal{T}(u)$ the corresponding series in $\mathrm{A}(\bar{R})\left[\left[u^{ \pm 1}\right]\right]$ and End $\mathbb{C}^{N} \otimes \mathrm{A}(\bar{R})\left[\left[u^{ \pm 1}\right]\right]$. Defining relations for the quantum current algebra,

$$
\bar{\tau}_{i j k l}^{(r, s)}-\underline{\tau}_{i j k l}^{(r, s)}=0, \quad \text { where } \quad i, j, k, l=1, \ldots, N \quad \text { and } \quad r, s \in \mathbb{Z},
$$

can be expressed as a commutation relation in End $\mathbb{C}^{N} \otimes$ End $\mathbb{C}^{N} \otimes \mathrm{A}(\bar{R})\left[\left[u^{ \pm 1}, v^{ \pm 1}\right]\right]$,

$$
\mathcal{T}_{1}(u) \bar{R}(u-v+h C)^{-1} \mathcal{T}_{2}(v) \bar{R}(u-v)=\bar{R}(-v+u)^{-1} \mathcal{T}_{2}(v) \bar{R}(-v+u-h C) \mathcal{T}_{1}(u)
$$

Since the images of the elements $\bar{\tau}_{i j k l}^{(r, s)}$ and $\underline{\tau}_{i j k l}^{(r, s)}$ in quotient (2.6) coincide, we denote them by $\tau_{i j k l}^{(r, s)}$. We also write $\mathcal{T}_{[1]}(u)=\mathcal{T}(u)$ and

$$
\mathcal{T}_{[2]}(u, v)=\sum_{i, j, k, l=1}^{N} \sum_{r, s \in \mathbb{Z}} e_{i j} \otimes e_{k l} \otimes \tau_{i j k l}^{(r, s)} u^{-r-1} v^{-s-1} \in\left(\text { End } \mathbb{C}^{N}\right)^{\otimes 2} \otimes \mathrm{A}(\bar{R})\left[\left[u^{ \pm 1}, v^{ \pm 1}\right]\right],
$$

so that, in particular, the both sides of commutation relation (2.8) coincide with $\mathcal{T}_{[2]}(u, v)$. 
Denote by $\mathrm{I}_{n}^{h}(\bar{R})$ and $\mathrm{I}_{n}(\bar{R})$ the images of the left ideals $\mathrm{I}_{n}^{h}(N)$ and $\mathrm{I}_{n}(N)$ in the algebra $\mathrm{A}(\bar{R})$ with respect to the canonical map $\widetilde{\mathrm{F}}(N) \rightarrow \mathrm{A}(\bar{R})$.

Corollary 2.3. For any $n \geqslant 1$ the element $\mathcal{T}_{[2]}(u, v)$ modulo $\mathrm{I}_{n}^{h}(\bar{R})$ belongs to

$$
\text { End } \mathbb{C}^{N} \otimes \operatorname{End} \mathbb{C}^{N} \otimes \mathrm{A}(\bar{R})((u, v)) \text {. }
$$

Proof. By Lemma 2.1, the left hand side of commutation relation (2.8) modulo $\mathrm{I}_{n}^{h}(\bar{R})$ belongs to $\left(\text { End } \mathbb{C}^{N}\right)^{\otimes 2} \otimes \mathrm{A}(\bar{R})\left[\left[u^{ \pm 1}\right]\right]((v))$ while the right hand side of (2.8) modulo $\mathrm{I}_{n}^{h}(\bar{R})$ belongs to $\left(\text { End } \mathbb{C}^{N}\right)^{\otimes 2} \otimes \mathrm{A}(\bar{R})\left[\left[v^{ \pm 1}\right]\right]((u))$. Hence, both sides of $(2.8)$ possess only finitely many negative powers of the variables $u$ and $v$ modulo $\mathrm{I}_{n}^{h}(\bar{R})$, so the corollary follows.

For any integer $n \geqslant 2$ and complex number $a$ introduce the functions with values in $\left(\text { End } \mathbb{C}^{N}\right)^{\otimes n}$ in the variables $u=\left(u_{1}, \ldots, u_{n}\right)$ by

$$
\begin{aligned}
& \bar{R}_{[n, a]}(u)=\prod_{i=1, \ldots, n-1} \prod_{j=i+1, \ldots, n} \bar{R}_{i j}\left(u_{i}-u_{j}+a h\right), \\
& \stackrel{\leftarrow}{R}_{[n, a]}(u)=\prod_{i=1, \ldots, n-1} \prod_{j=i+1, \ldots, n} \bar{R}_{i j}\left(u_{i}-u_{j}+a h\right),
\end{aligned}
$$

where the arrows indicate the order of the factors. If $a=0$, we omit the second subscript and write $\bar{R}_{[n]}(u)=\bar{R}_{[n, 0]}(u)$ and $\overleftarrow{\leftarrow}_{[n]}(u)=\overleftarrow{\leftarrow}_{[n, 0]}(u)$. The functions $R_{[n, a]}(u)$ and $\stackrel{\leftarrow}{R}_{[n, a]}(u)$ associated with Yang $R$-matrix (1.3) can be defined analogously. Introduce the expression

$$
\mathcal{T}_{[n]}(u)=\prod_{i=1, \ldots, n}\left(\mathcal{T}_{i}\left(u_{i}\right) \bar{R}_{i i+1}\left(u_{i}-u_{i+1}+h C\right)^{-1} \ldots \bar{R}_{i n}\left(u_{i}-u_{n}+h C\right)^{-1}\right) \cdot \bar{\leftarrow}_{[n]}(u) .
$$

For example, by setting $\widehat{R}_{i j}=\bar{R}_{i j}\left(u_{i}-u_{j}+h C\right)^{-1}, \bar{R}_{i j}=\bar{R}_{i j}\left(u_{i}-u_{j}\right)$ and $n=4$, we get

$$
\mathcal{T}_{[4]}(u)=\mathcal{T}_{1}\left(u_{1}\right) \widehat{R}_{12} \widehat{R}_{13} \widehat{R}_{14} \mathcal{T}_{2}\left(u_{2}\right) \widehat{R}_{23} \widehat{R}_{24} \mathcal{T}_{3}\left(u_{3}\right) \widehat{R}_{34} \mathcal{T}_{4}\left(u_{4}\right) \bar{R}_{34} \bar{R}_{24} \bar{R}_{23} \bar{R}_{14} \bar{R}_{13} \bar{R}_{12} .
$$

Proposition 2.4. For any $m \geqslant 1$ the expression $\mathcal{T}_{[m]}(u)$ is a well-defined element of

$$
\left(\text { End } \mathbb{C}^{N}\right)^{\otimes m} \otimes \mathrm{A}(\bar{R})\left[\left[u_{1}^{ \pm 1}, \ldots, u_{m}^{ \pm 1}\right]\right] .
$$

Moreover, for any integer $n \geqslant 1$ the element $\mathcal{T}_{[m]}(u)$ modulo $\mathrm{I}_{n}^{h}(\bar{R})$ belongs to

$$
\left(\text { End } \mathbb{C}^{N}\right)^{\otimes m} \otimes \mathrm{A}(\bar{R})\left(\left(u_{1}, \ldots, u_{m}\right)\right) .
$$

Proof. Fix an integer $n \geqslant 1$. The proposition follows by induction on $m$. The case $m=1$ is clear (and the case $m=2$ is already given by Corollary 2.3). Assume that the proposition holds for some integer $m \geqslant 1$. Definition in (2.12) implies

$$
\mathcal{T}_{[m+1]}(u, v)=\mathcal{T}_{[1]}^{13}(u) \bar{R}_{1 m}^{12}(u+h C \mid v)^{-1} \mathcal{T}_{[m]}^{23}(v) \bar{R}_{1 m}^{12}(u \mid v),
$$

where $v=\left(v_{1}, \ldots, v_{m}\right), u$ is a single variable and, as explained in Section 1.3, the superscripts indicate tensor copies in

$$
\underbrace{\operatorname{End} \mathbb{C}^{N}}_{1} \otimes \underbrace{\left(\operatorname{End} \mathbb{C}^{N}\right)^{\otimes m}}_{2} \otimes \underbrace{\mathrm{A}(\bar{R})}_{3}
$$


By arguing as in the proof of Lemma 2.1, one can prove that (2.13) is a well-defined element of

$$
\text { End } \mathbb{C}^{N} \otimes\left(\operatorname{End} \mathbb{C}^{N}\right)^{\otimes m} \otimes \mathrm{A}(\bar{R})\left[\left[u^{ \pm 1}, v_{1}^{ \pm 1}, \ldots, v_{m}^{ \pm 1}\right]\right] .
$$

As for the second assertion, by using Yang-Baxter equation (1.6) and commutation relation (2.8) one can prove the equality

$$
\begin{aligned}
\mathcal{T}_{[1]}^{13}(u) & \bar{R}_{1 m}^{12}(u+h C \mid v)^{-1} \mathcal{T}_{[m]}^{23}(v) \bar{R}_{1 m}^{12}(u \mid v) \\
= & \bar{R}_{1 m}^{12 r}(u \mid v)^{-1} \mathcal{T}_{[m]}^{23}(v) \bar{R}_{1 m}^{12 r}(u-h C \mid v) \mathcal{T}_{[1]}^{13}(u) .
\end{aligned}
$$

The superscript $r$ in (2.14) indicates that the rational functions $\bar{R}_{1 m}^{12 r}(u \mid v)^{-1}=\bar{R}_{1 m}^{12}(u \mid v)^{-1}$ and $\bar{R}_{1 m}^{12 r}(u-h C \mid v)=\bar{R}_{1 m}^{12}(u-h C \mid v)$ should be expanded in nonnegative powers of the variable $u$, thus violating the expansion convention introduced in Section 1.3. More precisely, in terms of the aforementioned expansion convention, we have

$$
\begin{aligned}
& \bar{R}_{1 m}^{12 r}(u \mid v)^{-1}=\bar{R}_{12}\left(-v_{1}+u\right)^{-1} \ldots \bar{R}_{1 m+1}\left(-v_{m}+u\right)^{-1} \text { and } \\
& \bar{R}_{1 m}^{12 r}(u-h C \mid v)=\bar{R}_{1 m+1}\left(-v_{m}+u-h C\right) \ldots \bar{R}_{12}\left(-v_{1}+u-h C\right),
\end{aligned}
$$

while, by definition in (1.14), we have

$$
\begin{aligned}
& \bar{R}_{1 m}^{12}(u \mid v)^{-1}=\bar{R}_{12}\left(u-v_{1}\right)^{-1} \ldots \bar{R}_{1 m+1}\left(u-v_{m}\right)^{-1} \text { and } \\
& \bar{R}_{1 m}^{12}(u-h C \mid v)=\bar{R}_{1 m+1}\left(u-v_{m}-h C\right) \ldots \bar{R}_{12}\left(u-v_{1}-h C\right) .
\end{aligned}
$$

Since the left hand side of (2.14) coincides with $\mathcal{T}_{[m+1]}(u, v)$, we may now proceed as in the proof of Corollary 2.3. More precisely, the right hand side of (2.14) modulo $\mathrm{I}_{n}^{h}(\bar{R})$ possesses finitely many negative powers of the variable $u$. By the induction hypothesis, the left hand side of (2.14) modulo $\mathrm{I}_{n}^{h}(\bar{R})$ possesses finitely many negative powers of the variables $v_{1}, \ldots, v_{m}$. Hence, $\mathcal{T}_{[m+1]}(u, v)$ modulo $\mathrm{I}_{n}^{h}(\bar{R})$ belongs to

$$
\text { End } \mathbb{C}^{N} \otimes\left(\text { End } \mathbb{C}^{N}\right)^{\otimes m} \otimes \mathrm{A}(\bar{R})\left(\left(u, v_{1}, \ldots, v_{m}\right)\right),
$$

as required.

By Proposition 2.4, for any integer $n \geqslant 1$ there exist elements $\tau_{i_{1} j_{1} \ldots i_{n} j_{n}}^{\left(r_{1}, \ldots, r_{n}\right)}$ in $\mathrm{A}(\bar{R})$, where $i_{1}, j_{1}, \ldots, i_{n}, j_{n}=1, \ldots, N$ and $r_{1}, \ldots, r_{n} \in \mathbb{Z}$, such that

$$
\mathcal{T}_{[n]}(u)=\sum_{i_{1}, j_{1}, \ldots, i_{n}, j_{n}=1}^{N} \sum_{r_{1}, \ldots, r_{n} \in \mathbb{Z}} e_{i_{1} j_{1}} \otimes \ldots \otimes e_{i_{n} j_{n}} \otimes \tau_{i_{1} j_{1} \ldots, i_{n} j_{n}}^{\left(r_{1}, \ldots, r_{n}\right)} u_{1}^{-r_{1}-1} \ldots u_{n}^{-r_{n}-1} .
$$

For any $a \in h \mathbb{C}[h, C]$ and the variables $u=\left(u_{1}, \ldots, u_{n}\right)$ denote by $u+a$ the shifted variables $\left(u_{1}+a, \ldots, u_{n}+a\right)$. We now employ elements (2.15) to write the more general form of commutation relation $(2.8)$ in

$$
\left(\text { End } \mathbb{C}^{N}\right)^{\otimes n} \otimes\left(\text { End } \mathbb{C}^{N}\right)^{\otimes m} \otimes \mathrm{A}(\bar{R})\left[\left[u_{1}^{ \pm 1}, \ldots, u_{n}^{ \pm 1}, v_{1}^{ \pm 1}, \ldots, v_{m}^{ \pm 1}\right]\right] .
$$

Proposition 2.5. For any integers $n, m \geqslant 1$ and the variables $u=\left(u_{1}, \ldots, u_{n}\right)$ and $v=\left(v_{1}, \ldots, v_{m}\right)$ we have

$$
\begin{aligned}
& \mathcal{T}_{[n+m]}(u, v)=\mathcal{T}_{[n]}^{13}(u) \bar{R}_{n m}^{12}(u+h C \mid v)^{-1} \mathcal{T}_{[m]}^{23}(v) \bar{R}_{n m}^{12}(u \mid v), \\
& \mathcal{T}_{[n+m]}(u, v)=\bar{R}_{n m}^{12 r}(u \mid v)^{-1} \mathcal{T}_{[m]}^{23}(v) \bar{R}_{n m}^{12 r}(u-h C \mid v) \mathcal{T}_{[n]}^{13}(u) .
\end{aligned}
$$


The superscript $r$ in (2.17) indicates that the rational functions $\bar{R}_{n m}^{12 r}(u \mid v)^{-1}=\bar{R}_{n m}^{12}(u \mid v)^{-1}$ and $\bar{R}_{n m}^{12 r}(u-h C \mid v)=\bar{R}_{n m}^{12}(u-h C \mid v)$ are expanded in nonnegative powers of the variables $u=\left(u_{1}, \ldots, u_{n}\right)$.

Proof. It is clear from definition in (2.12) that (2.16) holds for any integers $n, m \geqslant 1$. Moreover, (2.17) holds for $n=1$; recall (2.14). In general, for a fixed integer $m \geqslant 1$, one can prove by induction on $n$, which relies on (2.14) and Yang-Baxter equation (1.6), that the right hand sides of (2.16) and (2.17) coincide.

2.3. Quantum current algebra at the level $c$. For any $c \in \mathbb{C}$ define the quantum current algebra at the level $c$ as the quotient $\mathrm{A}_{c}(\bar{R})$ of the algebra $\mathrm{A}(\bar{R})$ by the ideal generated by $C-c$. We now use the vacuum module over the double Yangian at the level $c$, as defined in Section 1.3, to obtain an example of an $\mathrm{A}_{c}(\bar{R})$-module.

Proposition 2.6. For any $c \in \mathbb{C}$ the assignments

$$
\mathcal{T}_{[n]}(u) \mapsto T_{[n]}^{+}(u) T_{[n]}(u+h c / 2)^{-1}
$$

with $n \geqslant 1$ and the variables $u=\left(u_{1}, \ldots, u_{n}\right)$ define a structure of $\mathrm{A}_{c}(\bar{R})$-module on the vacuum module $\mathcal{V}_{c}\left(\mathfrak{g l}_{N}\right)$. In particular, the assignments

$$
\mathcal{T}_{[n]}(u) \mapsto T_{[n]}^{+}(u) \mathbf{1}
$$

with $n \geqslant 1$ define a $\mathbb{C}[[h]]$-module epimorphism

$$
\mathrm{A}_{c}(\bar{R}) \rightarrow \mathcal{V}_{c}\left(\mathfrak{g l}_{N}\right)
$$

Proof. Let us prove that the operator $T^{+}(u) T(u+h c / 2)^{-1}$ on $\mathcal{V}_{c}\left(\mathfrak{g l}_{N}\right)$ satisfies commutation equation $(2.8)$ at the level $c$. Consider the expression

$$
T_{1}^{+}(u) T_{1}(u+h c / 2)^{-1} \bar{R}(u-v+h c)^{-1} T_{2}^{+}(v) T_{2}(v+h c / 2)^{-1} \bar{R}(u-v),
$$

which corresponds to the left hand side of (2.8). Due to (1.11), this equals to

$$
T_{1}^{+}(u) T_{2}^{+}(v) \bar{R}(u-v)^{-1} T_{1}(u+h c / 2)^{-1} T_{2}(v+h c / 2)^{-1} \bar{R}(u-v) .
$$

Next, by using (1.9) and then canceling the $R$-matrices $\bar{R}(u-v)^{ \pm 1}$ we get

$$
T_{1}^{+}(u) T_{2}^{+}(v) T_{2}(v+h c / 2)^{-1} T_{1}(u+h c / 2)^{-1} .
$$

Consider the expression

$$
\bar{R}(-v+u)^{-1} T_{2}^{+}(v) T_{2}(v+h c / 2)^{-1} \bar{R}(-v+u-h c) T_{1}^{+}(u) T_{1}(u+h c / 2)^{-1},
$$

which corresponds to the right hand side of (2.8). Due to unitarity property (1.5), the $R$-matrix $\bar{R}(-v+u-h c)$ is equal to $\bar{R}(v-u+h c)^{-1}$, so we can employ (1.11) and write the given expression as

$$
\bar{R}(-v+u)^{-1} T_{2}^{+}(v) T_{1}^{+}(u) \bar{R}(v-u)^{-1} T_{2}(v+h c / 2)^{-1} T_{1}(u+h c / 2)^{-1} .
$$

Finally, by using (1.10) and then canceling the $R$-matrices $\bar{R}(v-u)^{-1}=\bar{R}(-v+u)$ and $\bar{R}(-v+u)^{-1}$ we obtain $(2.22)$.

Since (2.21) and (2.23) coincide, the assignment $\mathcal{T}(u) \mapsto T^{+}(u) T(u+h c / 2)^{-1}$ defines an $\mathrm{A}_{c}(\bar{R})$-module structure on $\mathcal{V}_{c}\left(\mathfrak{g l}_{N}\right)$. Our previous calculation also shows that the action 
of $\mathcal{T}_{[2]}(u)$ on $\mathcal{V}_{c}\left(\mathfrak{g l}_{N}\right)$ is given by $T_{[2]}^{+}(u) T_{[2]}(u+h c / 2)^{-1}$, i.e. that (2.18) holds for $n=2$ as well. In general, (2.18) with $n>2$ can be verified by induction on $n$, which relies on defining relations (1.9)-(1.11) for the double Yangian.

As for the second statement, observe that $T(u) \mathbf{1}=\mathbf{1}$, so the composition of map (2.18) and the evaluation map End $\mathcal{V}_{c}\left(\mathfrak{g l}_{N}\right) \ni a \mapsto a \cdot \mathbf{1} \in \mathcal{V}_{c}\left(\mathfrak{g l}_{N}\right)$ coincides with $\mathbb{C}[[h]]$-module map (2.20). Finally, surjectivity follows from the Poincaré-Birkhoff-Witt theorem for the double Yangian; see [9, Theorem 2.2].

Remark 2.7. Action (2.18) of the algebra $\mathrm{A}_{c}(\bar{R})$ possesses the form of the quantum currents from [27]; see also $[4,8]$. It suggests, together with some other results of the paper, a possibility of establishing a closer connection between the quantum current algebra and the (completed) double Yangian for the Lie algebra $\mathfrak{g l}_{N}$.

Let $A_{1}^{c} \subset \mathrm{A}_{c}(\bar{R})$ be the set which contains the unit 1 and all monomials $\tau_{i_{1} j_{1}}^{\left(r_{1}\right)} \ldots \tau_{i_{n} j_{n}}^{\left(r_{n}\right)}, \quad$ where $r_{1}, \ldots, r_{n} \in \mathbb{Z}, i_{1}, \ldots, i_{n}, j_{1}, \ldots, j_{n}=1, \ldots, N, n=1,2, \ldots$

Next, let $A_{2}^{c} \subset \mathrm{A}_{c}(\bar{R})$ be the set which contains the unit 1 and all elements $\tau_{i_{1} j_{1} \ldots i_{n} j_{n}}^{\left(r_{1}, \ldots, r_{n}\right)}, \quad$ where $\quad r_{1}, \ldots, r_{n} \in \mathbb{Z}, i_{1}, \ldots, i_{n}, j_{1}, \ldots, j_{n}=1, \ldots, N, n=1,2, \ldots$

Throughout this paper, span $A$ will always denote the linear span of a subset $A$ of some $\mathbb{C}[[h]]$-module, with respect to the ring $\mathbb{C}[[h]]$. For any subset $A$ of the algebra $\mathrm{A}_{c}(\bar{R})$ set

$$
[A]=\left\{a \in \mathrm{A}_{c}(\bar{R}): h^{n} a \in A \text { for some } n \geqslant 0\right\} .
$$

We denote the image of the left ideal $\mathrm{I}_{p}^{h}(\bar{R}) \subset \mathrm{A}(\bar{R})$ with respect to the canonical map $\mathrm{A}(\bar{R}) \rightarrow \mathrm{A}_{c}(\bar{R})$ by $\mathrm{I}_{p}^{h}(\bar{R})$

Proposition 2.8. Let $i=1,2$. For any integer $p \geqslant 1$ and element $a \in \mathrm{A}_{c}(\bar{R})$ there exist $a_{i} \in\left[\operatorname{span} A_{i}^{c}\right]$ such that $a-a_{i}$ belongs to $\mathrm{I}_{p}^{h}(\bar{R})$.

Proof. Fix an integer $p \geqslant 1$. Let $a$ be an arbitrary element of $\mathrm{A}_{c}(\bar{R})$. Denote by $B_{1}$ the subset of $\mathrm{F}(N)$ which contains the unit $1 \in \mathrm{F}(N)$ and all monomials $\tau_{i_{1} j_{1}}^{\left(r_{1}\right)} \ldots \tau_{i_{n} j_{n}}^{\left(r_{n}\right)} \in \mathrm{F}(N)$, where $r_{1}, \ldots, r_{n} \in \mathbb{Z}, i_{1}, \ldots, i_{n}, j_{1}, \ldots, j_{n}=1, \ldots, N, n=1,2, \ldots$ Choose any element $b$ in $\widetilde{\mathrm{F}}(N)$ such that its image in the algebra $\mathrm{A}_{c}(\bar{R})$, with respect to the canonical map $\pi_{c}: \widetilde{\mathrm{F}}(N) \rightarrow \mathrm{A}_{c}(\bar{R})$, is equal to $a$. There exist an element $b_{1}$ in

$$
\left[\operatorname{span} B_{1}\right]=\left\{x \in \widetilde{\mathrm{F}}(N): h^{n} x \in \operatorname{span} B_{1} \text { for some } n \geqslant 0\right\}
$$

such that $b-b_{1}$ belongs to $\mathrm{I}_{p}^{h}(N)$. Clearly, its image $a_{1}=\pi_{c}\left(b_{1}\right)$ belongs to $\left[\operatorname{span} A_{1}^{c}\right]$ and satisfies $a-a_{1} \in \mathrm{I}_{p}^{h}(\bar{R})$.

For any $n \geqslant 1$ and the variables $u=\left(u_{1}, \ldots, u_{n}\right)$ the element $\mathcal{T}_{[n]}(u)$ can be written as

$$
\mathcal{T}_{[n]}(u)=\left(\left(\overleftarrow{\leftarrow}_{[n, C]}(u)\right)_{\mathrm{RL}}^{-1} \cdot\left(\mathcal{T}_{1}\left(u_{1}\right) \ldots \mathcal{T}_{n}\left(u_{n}\right)\right)\right) \cdot \overleftarrow{\leftarrow}_{[n]}(u) .
$$

By using unitarity property (1.5) and crossing symmetry property (1.8) we can move all $R$-matrices in (2.24) to the left hand side, thus getting

$$
\stackrel{\leftarrow}{R}_{[n, C+N]}(u) \underset{\mathrm{RL}}{\cdot}\left(\mathcal{T}_{[n]}(u) \cdot\left(\stackrel{\leftarrow}{R}_{[n]}(u)\right)^{-1}\right)=\mathcal{T}_{1}\left(u_{1}\right) \ldots \mathcal{T}_{n}\left(u_{n}\right) .
$$


The elements of the set $A_{1}^{c}$ are exactly the coefficients, with respect to the variables $u_{1}, \ldots, u_{n}$ (and parameter $h$ ), of the matrix entries of $\mathcal{T}_{1}\left(u_{1}\right) \ldots \mathcal{T}_{n}\left(u_{n}\right)$ while the elements of the set $A_{2}^{c}$ are exactly the coefficients, with respect to the variables $u_{1}, \ldots, u_{n}$, of the matrix entries of $\mathcal{T}_{[n]}(u)$. Therefore, (2.25) implies that there exist $a_{2} \in\left[\operatorname{span} A_{2}^{c}\right]$ such that $a_{1}-a_{2}$ belongs to $\mathrm{I}_{p}^{h}(\bar{R})$. Finally, we obtain

$$
a-a_{2}=\left(a-a_{1}\right)+\left(a_{1}-a_{2}\right) \in \mathrm{I}_{p}^{h}(\bar{R}),
$$

as required.

For the variables $u=\left(u_{1}, \ldots, u_{n}\right)$ and an integer $i=1, \ldots, n-1$ set

$$
u_{i \leftrightarrow i+1}=\left(u_{1}, \ldots, u_{i-1}, u_{i+1}, u_{i}, u_{i+2}, \ldots, u_{n}\right) \quad \text { and } \quad \overleftarrow{u}=\left(u_{n}, \ldots, u_{1}\right) .
$$

We use the following lemma in the proof of Theorem 3.3.

Lemma 2.9. For any integer $n \geqslant 2$ and the variables $u=\left(u_{1}, \ldots, u_{n}\right)$ we have

$$
\bar{R}_{i i+1}\left(u_{i}-u_{i+1}\right) \mathcal{T}_{[n]}(u) \bar{R}_{i i+1}\left(u_{i}-u_{i+1}\right)^{-1}=P_{i i+1} \mathcal{T}_{[n]}\left(u_{i \leftrightarrow i+1}\right) P_{i i+1} .
$$

In particular, for $n=2$ we have $u=\left(u_{1}, u_{2}\right)$ and

$$
\bar{R}\left(u_{1}-u_{2}\right) \mathcal{T}_{[2]}\left(u_{1}, u_{2}\right) \bar{R}\left(u_{1}-u_{2}\right)^{-1}=P \mathcal{T}_{[2]}\left(u_{2}, u_{1}\right) P .
$$

Proof. Equality (2.27) follows from unitarity property (1.5) and relation (2.8). Finally, by using (2.27) and Yang-Baxter equation (1.6) one can easily verify (2.26).

Remark 2.10. One can show by a short calculation that commutation relation (2.8) can be written as $h^{2} X=0$, where $X$ belongs to $\left(\text { End } \mathbb{C}^{N}\right)^{\otimes 2} \otimes \mathrm{A}_{c}(\bar{R})\left[\left[u^{ \pm 1}, v^{ \pm 1}\right]\right]$. Due to Proposition 2.2, the algebra $\mathrm{A}_{c}(\bar{R})$ is torsion-free, so we have $X=0$. By considering the coefficients of the matrix entries $e_{i j} \otimes e_{k l}$ with respect to the variables $u^{-r-1} v^{-s-1}$ in the equality $\left.X\right|_{h=0}=0$, where $\left.X\right|_{h=0}$ denotes the evaluation of $X$ at $h=0$, we find

$$
\left[\tau_{i j}^{(r)}, \tau_{k l}^{(s)}\right]=\delta_{j k} \tau_{i l}^{(r+s)}-\delta_{i l} \tau_{k j}^{(r+s)}+\delta_{r+s 0} c r\left(\delta_{i l} \delta_{j k}-\frac{\delta_{i j} \delta_{k l}}{N}\right)
$$

i.e. the commutation relation for the affine Lie algebra $\widehat{\mathfrak{g l}}_{N}=\mathfrak{g l}_{N} \otimes \mathbb{C}\left[t, t^{-1}\right] \oplus \mathbb{C} C$ at the level $C=c$.

2.4. Central elements of the quantum current algebra at the critical level. This section presents a digression from the main topic as its results are not needed in the rest of the paper. Consider the following permutation operator on $\left(\mathbb{C}^{N}\right)^{\otimes n}$,

$$
P_{[n]}: x_{1} \otimes \ldots \otimes x_{n} \mapsto x_{n} \otimes \ldots \otimes x_{1} .
$$

Denote the element $P_{[n]} \mathcal{T}_{[n]}(\overleftarrow{u}) P_{[n]}$ more briefly by $\overleftarrow{\mathcal{T}}_{[n]}(u)$, so that, in particular, $(2.27)$ can be written in the form which resembles $R T T$ relations,

$$
\bar{R}\left(u_{1}-u_{2}\right) \mathcal{T}_{[2]}(u)=\overleftarrow{\mathcal{T}}_{[2]}(u) \bar{R}\left(u_{1}-u_{2}\right) .
$$

Recall notation (2.10)-(2.11). By combining Lemma 2.9 and Yang-Baxter equation (1.6) one can generalize (2.28) as follows.

Lemma 2.11. For any integer $n \geqslant 2$ and the variables $u=\left(u_{1}, \ldots, u_{n}\right)$ we have

$$
R_{[n]}(u) \mathcal{T}_{[n]}(u)=\overleftarrow{\mathcal{T}}_{[n]}(u) R_{[n]}(u)
$$


We now recall a special version of the fusion procedure for Yang $R$-matrix (1.3) originated in [11]; see also [24, Section 6.4] for more details and references. The symmetric group $\mathfrak{S}_{n}$ acts on the space $\left(\mathbb{C}^{N}\right)^{\otimes n}$ by permuting the tensor factors. Denote by $A^{(n)}$ the action of the anti-symmetrizer

$$
a^{(n)}=\frac{1}{n !} \sum_{s \in \mathfrak{S}_{n}} \operatorname{sgn} s \cdot s \in \mathbb{C}\left[\mathfrak{S}_{n}\right]
$$

on $\left(\mathbb{C}^{N}\right)^{\otimes n}$. Due to [11], the consecutive evaluations $u_{1}=0, u_{2}=-h, u_{3}=-2 h, \ldots, u_{n}=$ $-(n-1) h$ of the variables $u=\left(u_{1}, \ldots, u_{n}\right)$ in $R_{[n]}(u)$ are well-defined and we have

$$
\left.\left.\left.R_{[n]}(u)\right|_{u_{1}=0}\right|_{u_{2}=-h} \cdots\right|_{u_{n}=-(n-1) h}=n ! A^{(n)} .
$$

For the variable $u$ set $u_{[n]}=(u, u-h, \ldots, u-(n-1) h)$. The next two lemmas are used in the proof of Theorem 2.14.

Lemma 2.12. The following equalities hold on End $\mathbb{C}^{N} \otimes\left(\text { End } \mathbb{C}^{N}\right)^{\otimes n}$ :

$$
\begin{aligned}
A^{(n)} \bar{R}_{1 n}^{12}\left(u_{0} \mid u_{[n]}\right) & =\overleftarrow{\leftarrow}_{1 n}^{12}\left(u_{0} \mid u_{[n]}\right) A^{(n)}, \\
A^{(n)} \bar{R}_{1 n}^{12 r}\left(u_{0} \mid u_{[n]}\right) & =\overleftarrow{\leftarrow}_{1 n}^{12 r}\left(u_{0} \mid u_{[n]}\right) A^{(n)},
\end{aligned}
$$

where the tensor factors of End $\mathbb{C}^{N} \otimes\left(\text { End } \mathbb{C}^{N}\right)^{\otimes n}$ are labeled by $0,1, \ldots, n$ and $A^{(n)}$ is applied on the tensor factors $1, \ldots, n$, i.e. $A^{(n)}$ denotes the operator $1 \otimes A^{(n)}$ on End $\mathbb{C}^{N} \otimes$ $\left(\text { End } \mathbb{C}^{N}\right)^{\otimes n}$. The superscript $r$ in (2.32) indicates the rational functions $\bar{R}_{1 n}^{12 r}\left(u_{0} \mid u_{[n]}\right)=$ $\bar{R}_{1 n}^{12}\left(u_{0} \mid u_{[n]}\right)$ and $\overleftarrow{\leftarrow}_{1 n}^{12 r}\left(u_{0} \mid u_{[n]}\right)=\overleftarrow{\leftarrow}_{1 n}^{12}\left(u_{0} \mid u_{[n]}\right)$ are expanded in negative powers of the variable $u$.

Proof. The lemma follows directly from fusion procedure (2.30). More details on its proof can be found in, e.g., [14, Lemma 3.1].

Lemma 2.13. The following equality holds on $\left(\text { End } \mathbb{C}^{N}\right)^{\otimes n} \otimes \mathrm{A}_{-N}(\bar{R})$ :

$$
A^{(n)} \mathcal{T}_{[n]}\left(u_{[n]}\right)=\overleftarrow{\mathcal{T}}_{[n]}\left(u_{[n]}\right) A^{(n)}
$$

Proof. The lemma easily follows by applying the consecutive evaluations $u_{1}=0, u_{2}=$ $-h, \ldots, u_{n}=-(n-1) h$ on $(2.29)$ and employing fusion procedure (2.30).

We now consider the quantum current algebra at the critical level $c=-N$. For each $n=1, \ldots, N$ introduce the series

$$
\mathfrak{T}_{[n]}(u)=\operatorname{tr}_{1, \ldots, n} A^{(n)} \mathcal{T}_{[n]}\left(u_{[n]}\right) \in \mathrm{A}_{-N}(\bar{R})\left[\left[u^{ \pm 1}\right]\right],
$$

where the trace is taken over all $n$ copies of End $\mathbb{C}^{N}$. The proof of the next theorem is similar to the proof of [14, Theorem 3.2]. It relies on certain techniques whose $R T T$ counterparts are well-known; see, e.g., proof of [5, Theorem 3.2] or [9, Theorem 4.4], cf. also $[26,29]$.

Theorem 2.14. All coefficients of $\mathfrak{T}_{[n]}(u)$ belong to the center of the algebra $\mathrm{A}_{-N}(\bar{R})$.

Proof. We will prove the equality

$$
\mathcal{T}\left(u_{0}\right) \mathfrak{T}_{[n]}(u) \underset{16}{=} \mathfrak{T}_{[n]}(u) \mathcal{T}\left(u_{0}\right)
$$


in End $\mathbb{C}^{N} \otimes \mathrm{A}_{-N}(\bar{R})$, which implies the statement of the theorem. By applying $\mathcal{T}\left(u_{0}\right)$ on $(2.34)$ we get

$$
\operatorname{tr}_{1, \ldots, n} A^{(n)} \mathcal{T}_{0}\left(u_{0}\right) \mathcal{T}_{[n]}\left(u_{[n]}\right),
$$

where the expression under the trace belongs to End $\mathbb{C}^{N} \otimes\left(\text { End } \mathbb{C}^{N}\right)^{\otimes n} \otimes \mathrm{A}_{-N}(\bar{R})$. The copies of End $\mathbb{C}^{N}$ in $(2.36)$ are labeled by $0,1, \ldots, n$. The series $\mathcal{T}\left(u_{0}\right)$ is applied on the tensor factor 0 while $A^{(n)}$ and $\mathcal{T}_{[n]}\left(u_{[n]}\right)$ are applied on the tensor factors $1, \ldots, n$. Due to the equality of the right hand sides in (2.16) and in (2.17), we can use crossing symmetry property (1.8) to express (2.36) as

$$
\begin{aligned}
& \operatorname{tr}_{1, \ldots, n} A^{(n)} A_{\mathrm{RL}}\left(\left(C \mathcal{T}_{[n]}\left(u_{[n]}\right) D \mathcal{T}_{0}\left(u_{0}\right)\right) \cdot B\right), \quad \text { where } \quad A=\bar{R}_{1 n}^{12}\left(u_{0} \mid u_{[n]}\right), \\
& B=\bar{R}_{1 n}^{12}\left(u_{0} \mid u_{[n]}\right)^{-1}, \quad C=\bar{R}_{1 n}^{12 r}\left(u_{0} \mid u_{[n]}\right)^{-1} \quad \text { and } \quad D=\bar{R}_{1 n}^{12 r}\left(u_{0}+h N \mid u_{[n]}\right) .
\end{aligned}
$$

Recall that the meaning of the superscript $\mathrm{r}$ is explained in the statement of Lemma 2.12. By (2.31) and (2.32) we have

$$
A^{(n)} Z=\overleftarrow{Z} A^{(n)} \quad \text { for } Z=A, B, C, D .
$$

Therefore, since $\left(A^{(n)}\right)^{2}=A^{(n)}$, we conclude that (2.37) is equal to

$$
\operatorname{tr}_{1, \ldots, n} \overleftarrow{A}_{\mathrm{RL}}\left(\left(\overleftarrow{C}\left(A^{(n)}\right)^{2} \mathcal{T}_{[n]}\left(u_{[n]}\right) D \mathcal{T}_{0}\left(u_{0}\right)\right) \cdot B\right)
$$

Next, we employ (2.33) and (2.38) to move one copy of $A^{(n)}$ to the left and another copy of $A^{(n)}$ to the right, thus getting

$$
\operatorname{tr}_{1, \ldots, n} A^{(n)} A_{\mathrm{RL}} \cdot\left(\left(C \stackrel{\leftarrow}{\mathcal{T}_{[n]}}\left(u_{[n]}\right) \stackrel{\leftarrow}{D} \mathcal{T}_{0}\left(u_{0}\right)\right) \cdot \stackrel{\leftarrow}{B} A^{(n)}\right)
$$

By the cyclic property of the trace and $\left(A^{(n)}\right)^{2}=A^{(n)}$ this equals to

$$
\operatorname{tr}_{1, \ldots, n} A_{\mathrm{RL}} \cdot\left(\left(C \stackrel{\leftarrow}{\mathcal{T}}_{[n]}\left(u_{[n]}\right) \stackrel{\leftarrow}{D} \mathcal{T}_{0}\left(u_{0}\right)\right) \cdot \stackrel{\leftarrow}{B} A^{(n)}\right)
$$

Finally, using (2.33) and (2.38) we move the remaining copy of $A^{(n)}$ to the left:

$$
\operatorname{tr}_{1, \ldots, n} A_{\mathrm{RL}}\left(\left(C A^{(n)} \mathcal{T}_{[n]}\left(u_{[n]}\right) D \mathcal{T}_{0}\left(u_{0}\right)\right) \cdot B\right) .
$$

Since $B A=1$, by employing the cyclic property of the trace and moving the tensor factors $1, \ldots, n$ of $A$ to the right we obtain

$$
\operatorname{tr}_{1, \ldots, n}\left(C A^{(n)} \mathcal{T}_{[n]}\left(u_{[n]}\right) D \mathcal{T}_{0}\left(u_{0}\right)\right) \cdot(B A)=\operatorname{tr}_{1, \ldots, n} C A^{(n)} \mathcal{T}_{[n]}\left(u_{[n]}\right) D \mathcal{T}_{0}\left(u_{0}\right)
$$

In order to finish the proof, i.e. to verify (2.35), it is sufficient to check that

$$
\operatorname{tr}_{1, \ldots, n} C A^{(n)} \mathcal{T}_{[n]}\left(u_{[n]}\right) D
$$

is equal to $\mathfrak{T}_{[n]}(u)$. However, crossing symmetry property $(1.8)$ implies $C_{\mathrm{LR}} \cdot D=1$. Hence, using the cyclic property of the trace and moving the tensor factors $1, \ldots, n$ of $C$ to the right, we rewrite (2.39) as follows:

$$
\begin{aligned}
& \operatorname{tr}_{1, \ldots, n} C A^{(n)} \mathcal{T}_{[n]}\left(u_{[n]}\right) D=\operatorname{tr}_{1, \ldots, n} C_{\mathrm{LR}} \cdot\left(A^{(n)} \mathcal{T}_{[n]}\left(u_{[n]}\right) D\right) \\
= & \operatorname{tr}_{1, \ldots, n}\left(A^{(n)} \mathcal{T}_{[n]}\left(u_{[n]}\right)\right) \cdot\left(C_{\mathrm{LR}} \cdot D\right)=\operatorname{tr}_{1, \ldots, n} A^{(n)} \mathcal{T}_{[n]}\left(u_{[n]}\right)=\mathfrak{T}_{[n]}(u),
\end{aligned}
$$

thus proving the theorem. 
Remark 2.15. Recall action (2.18) of the quantum current algebra on the vacuum module. It is worth noting that the action of $\mathfrak{T}_{[n]}(u)$ coincides with the action of certain Laurent series $\widetilde{\mathbb{T}}_{1^{n}}(u)$, whose coefficients belong to the center of the completed double Yangian $\widetilde{\mathrm{DY}}_{-N}\left(\mathfrak{g l}_{N}\right)$ at the critical level $c=-N$; see [9, Theorem 4.4] for more details.

Remark 2.16. In [2, Proposition 3.1], Ding found a realization of the quantum affine algebra $U_{q}\left(\widehat{\mathfrak{g l}}_{N}\right)$ via commutation relation of the form (2.8), which involves trigonometric $R$-matrix. The proof of Theorem 2.14 and fusion procedure from [1] suggest that the analogous construction of central elements of the completed quantum affine algebra $U_{q}\left(\widehat{\mathfrak{g l}}_{N}\right)$ at the critical level might be given in terms of Ding's realization, as long as the corresponding (trigonometric) $R$-matrix is appropriately normalized. The image of such family of central elements in the $R T T$ presentation of the completed quantum affine algebra $U_{q}\left(\widehat{\mathfrak{g l}}_{N}\right)$ at the critical level should coincide with the coefficients of the Laurent series $\ell_{k}(z), k=1, \ldots, N$, as defined in [5, Section 3], thus providing a new proof of [5, Theorem $3.2]$.

\section{Vacuum module over the quantum CURREnt Algebra}

In this section, we introduce the vacuum module $\mathcal{V}_{c}(\bar{R})$ for the quantum current algebra and we show that it is isomorphic, as a $\mathbb{C}[[h]]$-module, to the vacuum module over the double Yangian. This allows us to employ Etingof-Kazhdan's construction and obtain the structure of quantum vertex algebra on $\mathcal{V}_{c}(\bar{R})$. Next, in parallel with representation theory of the affine Lie algebras, we introduce the notion of restricted module for the algebra $\mathrm{A}(\bar{R})$. Finally, we prove that restricted level $c$ modules for the algebra $\mathrm{A}(\bar{R})$ are naturally equipped with a structure of module for the quantum vertex algebra $\mathcal{V}_{c}(\bar{R})$ and vice versa.

3.1. Properties of the vacuum module. Introduce the subset $W_{2}^{c}$ of $A_{2}^{c}$ by

$$
W_{2}^{c}=\left\{\tau_{i_{1} j_{1} \ldots i_{n} j_{n}}^{\left(r_{1}, \ldots, r_{n}\right)} \in A_{2}^{c}: r_{k} \geqslant 0 \text { for some } k=1, \ldots, n\right\} .
$$

Let $\mathrm{W}_{c}(\bar{R})$ be the left ideal in the algebra $\mathrm{A}_{c}(\bar{R})$ generated by the set $W_{2}^{c}$. Introduce the completion of $\mathrm{W}_{c}(\bar{R})$ as the inverse limit

$$
\mathrm{W}_{c}^{\prime}(\bar{R})=\lim _{\longleftarrow} \mathrm{W}_{c}(\bar{R}) / \mathrm{W}_{c}(\bar{R}) \cap \mathrm{I}_{p}(\bar{R}) .
$$

Then the $h$-adic completion $\left[\widetilde{\mathrm{W}_{c}^{\prime}(\bar{R})}\right]$ of

$$
\left[\mathrm{W}_{c}^{\prime}(\bar{R})\right]=\left\{a \in \mathrm{A}_{c}(\bar{R}): h^{n} a \in \mathrm{W}_{c}^{\prime}(\bar{R}) \text { for some } n \geqslant 0\right\}
$$

is also a left ideal in $\mathrm{A}_{c}(\bar{R})$. Define the vacuum module $\mathcal{V}_{c}(\bar{R})$ as the quotient of the algebra $\mathrm{A}_{c}(\bar{R})$ by its left ideal $\left[\widetilde{\mathrm{W}_{c}^{\prime}(\bar{R})}\right]$,

$$
\left.\mathcal{V}_{c}(\bar{R})=\mathrm{A}_{c}(\bar{R}) / \widetilde{\left[\mathrm{W}_{c}^{\prime}(\bar{R})\right.}\right] \text {. }
$$

Observe that the canonical map $\mathrm{A}(\bar{R}) \rightarrow \mathcal{V}_{c}(\bar{R})$ maps the left ideal $\mathrm{I}_{p}^{h}(\bar{R})$ to $h^{p} \mathcal{V}_{c}(\bar{R})$.

Proposition 3.1. The vacuum module $\mathcal{V}_{c}(\bar{R})$ is topologically free. 
Proof. The algebra $\mathrm{A}_{c}(\bar{R})$ is topologically free, so the proposition can be verified by arguing as in the proof of Proposition 2.2.

Denote the image of the unit $1 \in \mathrm{A}_{c}(\bar{R})$ in quotient (3.1) by $\mathbf{1}$. Let $V^{c}$ be the set of all elements $a \cdot \mathbf{1} \in \mathcal{V}_{c}(\bar{R})$ such that $a \in A_{2}^{c} \backslash W_{2}^{c}$, i.e.

$$
V^{c}=\{\mathbf{1}\} \cup\left\{\tau_{i_{1} j_{1} \ldots i_{n} j_{n}}^{\left(r_{1}, \ldots, r_{n}\right)} \mathbf{1}: \tau_{i_{1} j_{1} \ldots i_{n} j_{n}}^{\left(r_{1}, \ldots, r_{n}\right)} \in A_{2}^{c} \text { and } r_{k}<0 \text { for all } k=1, \ldots, n\right\} .
$$

As a direct consequence of Proposition 2.8 we obtain

Proposition 3.2. The $\mathbb{C}[[h]]$-module $\mathcal{V}_{c}(\bar{R})$ coincides with the $h$-adic completion of

$$
\left[\operatorname{span} V^{c}\right]=\left\{v \in \mathcal{V}_{c}(\bar{R}): h^{n} v \in \operatorname{span} V^{c} \text { for some } n \geqslant 0\right\} \text {. }
$$

We are now ready to prove the main result in this subsection.

Theorem 3.3. The assignments

$$
\mathcal{T}_{[n]}(u) \mathbf{1} \mapsto T_{[n]}^{+}(u) \mathbf{1}
$$

with $n \geqslant 1$ and the variables $u=\left(u_{1}, \ldots, u_{n}\right)$ define a $\mathbb{C}[[h]]$-module isomorphism

$$
\mathcal{V}_{c}(\bar{R}) \rightarrow \mathcal{V}_{c}\left(\mathfrak{g l}_{N}\right) \text {. }
$$

Proof. In order to prove that (3.2) defines a homomorphism of $\mathbb{C}[[h]]$-modules, it is sufficient to check that the elements of the set $W_{2}^{c}$ belong to the kernel of $\mathbb{C}[[h]]$-module map (2.20). Let $\tau=\tau_{i_{1} j_{1} \ldots i_{n} j_{n}}^{\left(r_{1}, \ldots, r_{n}\right)}$ be an arbitrary element of $W_{2}^{c}$. Then $r_{k} \geqslant 0$ for some $k=$ $1, \ldots, n$. The image $\widehat{\tau} \in \mathcal{V}_{c}\left(\mathfrak{g l}_{N}\right)$ of the element $\tau$, with respect to map (2.20), coincides with the coefficient of the variables $u_{1}^{-r_{1}-1} \ldots u_{n}^{-r_{n}-1}$ in the matrix entry $e_{i_{1} j_{1}} \otimes \ldots \otimes e_{i_{n} j_{n}}$ of the expression

$$
T_{[n]}^{+}(u) T_{[n]}(u+h c / 2)^{-1} \mathbf{1}=T_{[n]}^{+}(u) \mathbf{1} \in\left(\operatorname{End} \mathbb{C}^{N}\right)^{\otimes n} \otimes \mathcal{V}_{c}\left(\mathfrak{g l}_{N}\right)\left[\left[u_{1}, \ldots, u_{n}\right]\right] .
$$

Since (3.4) does not contain any negative powers of the variable $u_{k}$, we conclude that $\widehat{\tau}$ equals 0 , as required. Therefore, assignments (3.2) define a $\mathbb{C}[[h]]$-module homomorphism $\mathcal{V}_{c}(\bar{R}) \rightarrow \mathcal{V}_{c}\left(\mathfrak{g l}_{N}\right)$. Moreover, by the Poincaré-Birkhoff-Witt theorem for the double Yangian, see [9, Theorem 2.2], this map is surjective.

Let us prove that the assignments

$$
T_{[n]}^{+}(u) \mathbf{1} \mapsto \mathcal{T}_{[n]}(u) \mathbf{1}
$$

with $n \geqslant 1$ and the variables $u=\left(u_{1}, \ldots, u_{n}\right)$ define a $\mathbb{C}[[h]]$-module epimorphism

$$
\mathcal{V}_{c}\left(\mathfrak{g l}_{N}\right) \rightarrow \mathcal{V}_{c}(\bar{R})
$$

In order to verify that (3.5) defines a $\mathbb{C}[[h]]$-module homomorphism, it is sufficient to check that (3.5) maps the ideal of dual Yangian relations (1.10) to itself. However, this follows from Lemma 2.9. Indeed, by (1.10) we have

$$
\bar{R}_{i i+1}\left(u_{i}-u_{i+1}\right) T_{[n]}^{+}(u) \mathbf{1} \bar{R}_{i i+1}\left(u_{i}-u_{i+1}\right)^{-1}=P_{i i+1} T_{[n]}^{+}\left(u_{i \leftrightarrow i+1}\right) \mathbf{1} P_{i i+1}
$$

for any $i=1, \ldots, n-1$. The images of the left and the right hand side in (3.7), with respect to (3.5), are equal to the left and the right hand side in the equality

$$
\bar{R}_{i i+1}\left(u_{i}-u_{i+1}\right) \mathcal{T}_{[n]}(u) \mathbf{1} \bar{R}_{i i+1}\left(u_{i}-u_{i+1}\right)^{-1}=P_{i i+1} \mathcal{T}_{[n]}\left(u_{i \leftrightarrow i+1}\right) \mathbf{1} P_{i i+1},
$$


which follows by applying (2.26) on $\mathbf{1} \in \mathcal{V}_{c}(\bar{R})$. Hence, we conclude that (3.5) defines a $\mathbb{C}[[h]]$-module homomorphism. Moreover, the set $V^{c}$ is contained within the image of (3.6), so the map is surjective by Proposition 3.2. Finally, since maps (3.3) and (3.6) are inverses of each other, the theorem follows.

We now proceed towards the definition of restricted $\mathrm{A}(\bar{R})$-module, which is motivated by the following proposition.

Proposition 3.4. For any integer $n \geqslant 1$ and the variables $u=\left(u_{1}, \ldots, u_{n}\right)$ we have

$$
\mathcal{T}_{[n]}(u) w \in\left(\text { End } \mathbb{C}^{N}\right)^{\otimes n} \otimes \mathcal{V}_{c}(\bar{R})\left(\left(u_{1}, \ldots, u_{n}\right)\right)[[h]] \quad \text { for all } w \in \mathcal{V}_{c}(\bar{R}) .
$$

Proof. By applying (2.16) on the element $\mathbf{1} \in \mathcal{V}_{c}(\bar{R})$ we get

$$
\mathcal{T}_{[n+m]}(u, v) \mathbf{1}=\mathcal{T}_{[n]}^{13}(u) \bar{R}_{n m}^{12}(u+h c \mid v)^{-1} \mathcal{T}_{[m]}^{23}(v) \bar{R}_{n m}^{12}(u \mid v) \mathbf{1}
$$

for the variables $u=\left(u_{1}, \ldots, u_{n}\right)$ and $v=\left(v_{1}, \ldots, v_{m}\right)$. Note that this expression contains only nonnegative powers of the variables $u_{1}, \ldots, u_{n}$ and $v_{1}, \ldots, v_{m}$. Using crossing symmetry property (1.8) we move all $R$-matrices to the left hand side, thus getting

$$
\bar{R}_{n m}^{12}(u+h c+h N \mid v)_{\mathrm{RL}} \cdot\left(\mathcal{T}_{[n+m]}(u, v) \mathbf{1} \cdot \bar{R}_{n m}^{12}(u \mid v)^{-1}\right)=\mathcal{T}_{[n]}^{13}(u)\left(\mathcal{T}_{[m]}^{23}(v) \mathbf{1}\right) .
$$

Finally, we observe that, for any given integers $a_{1}, \ldots, a_{m} \geqslant 0$ and $p \geqslant 1$, the coefficient of the monomial $v_{1}^{a_{1}} \ldots v_{m}^{a_{m}}$ on left hand side of the given equation contains only finitely many negative powers of the variables $u_{1}, \ldots, u_{n}$ modulo $h^{p}$. Since the set of coefficients of the matrix entries of all $\mathcal{T}_{[m]}(v) \mathbf{1}$ with $m \geqslant 0$ coincides with $V^{c}$, the proposition follows from Proposition 3.2.

An $\mathrm{A}(\bar{R})$-module $W$ is said to be restricted if $W$ is a topologically free $\mathbb{C}[[h]]$-module such that

$$
\mathcal{T}(u) w \in \text { End } \mathbb{C}^{N} \otimes W((u))[[h]] \text { for all } w \in W .
$$

Also, as usual, an $\mathrm{A}(\bar{R})$-module $W$ is said to be a level $c$ module if the central element $C \in \mathrm{A}(\bar{R})$ acts on $W$ as a scalar multiplication by some $c \in \mathbb{C}$. Propositions 3.1 and 3.4 imply that the vacuum module $\mathcal{V}_{c}(\bar{R})$ is restricted $\mathrm{A}(\bar{R})$-module of level $c$.

Proposition 3.5. Let $W$ be a restricted $\mathrm{A}(\bar{R})$-module. Then

$$
\mathcal{T}_{[n]}(u) w \in\left(\text { End } \mathbb{C}^{N}\right)^{\otimes n} \otimes W\left(\left(u_{1}, \ldots, u_{n}\right)\right)[[h]] \text { for all } w \in W \text { and } n \geqslant 1 .
$$

Proof. The statement follows by induction on $n$ which is based on relations (2.16)-(2.17) and arguments from the proof of Proposition 2.4.

Suppose $W$ is a restricted $\mathrm{A}(\bar{R})$-module of level $c$. For the variable $z$ and the variables $u=\left(u_{1}, \ldots, u_{n}\right)$ introduce the elements of $\left(\text { End } \mathbb{C}^{N}\right)^{\otimes n} \otimes($ End $W)((z))\left[\left[u_{1}, \ldots, u_{n}, h\right]\right]$ by

$$
\mathcal{T}_{[n]}(u \mid z)=\prod_{i=1, \ldots, n}^{\longrightarrow}\left(\mathcal{T}_{i}\left(z+u_{i}\right) \bar{R}_{i i+1}\left(u_{i}-u_{i+1}+h c\right)^{-1} \ldots \bar{R}_{i n}\left(u_{i}-u_{n}+h c\right)^{-1}\right) \cdot \overleftarrow{\leftarrow}_{[n]}(u) .
$$

In particular, we have $\mathcal{T}_{[1]}(u \mid z)=\mathcal{T}(z+u)$. The following proposition is required in the proof of Theorem 3.9.

Proposition 3.6. Let $W$ be a restricted $\mathrm{A}(\bar{R})$-module of level $c$. 
(a) For any integers $n, m \geqslant 1$ the equalities

$$
\begin{aligned}
& \mathcal{T}_{[n+m]}\left(z_{1}+u_{1}, \ldots, z_{1}+u_{n}, z_{2}+v_{1}, \ldots, z_{2}+v_{m}\right) \\
= & \mathcal{T}_{[n]}^{13}\left(u \mid z_{1}\right) \bar{R}_{n m}^{12}\left(u|v| z_{1}-z_{2}+h c\right)^{-1} \mathcal{T}_{[m]}^{23}\left(v \mid z_{2}\right) \bar{R}_{n m}^{12}\left(u|v| z_{1}-z_{2}\right) \\
= & \bar{R}_{n m}^{12}\left(u|v|-z_{2}+z_{1}\right)^{-1} \mathcal{T}_{[m]}^{23}\left(v \mid z_{2}\right) \bar{R}_{n m}^{12}\left(u|v|-z_{2}+z_{1}-h c\right) \mathcal{T}_{[n]}^{13}\left(u \mid z_{1}\right)
\end{aligned}
$$

hold in

$$
\left(\text { End } \mathbb{C}^{N}\right)^{\otimes n} \otimes\left(\text { End } \mathbb{C}^{N}\right)^{\otimes m} \otimes(\text { End } W)\left(\left(z_{1}, z_{2}\right)\right)\left[\left[u_{1}, \ldots, u_{n}, v_{1}, \ldots, v_{m}, h\right]\right] .
$$

(b) For any $n \geqslant 2$ and $i=1, \ldots, n-1$ the equality

$$
\bar{R}_{i i+1}\left(u_{i}-u_{i+1}\right) \mathcal{T}_{[n]}(u \mid z) \bar{R}_{i i+1}\left(u_{i}-u_{i+1}\right)^{-1}=P_{i i+1} \mathcal{T}_{[n]}\left(u_{i \leftrightarrow i+1} \mid z\right) P_{i i+1}
$$

holds in $\left(\text { End } \mathbb{C}^{N}\right)^{\otimes n} \otimes($ End $W)\left(\left(z, u_{i}\right)\right)\left[\left[u_{1}, \ldots, u_{n}, h\right]\right]$.

Proof. Proposition 2.5 implies the first and Lemma 2.9 implies the second statement of the proposition.

3.2. Vacuum module as a quantum vertex algebra. From now on, we often identify the $\mathbb{C}[[h]]$-modules $\mathcal{V}_{c}(\bar{R})$ and $\mathcal{V}_{c}\left(\mathfrak{g l}_{N}\right)$ via the $\mathbb{C}[[h]]$-module isomorphism established in Theorem 3.3. For example, we utilize such identification in the next theorem, which is due to Etingof and Kazhdan; see [4, Theorem 2.3]. However, the vertex operator map in (3.11) is expressed somewhat differently from the original version in [4], so we demonstrate in the proof that both definitions coincide.

Theorem 3.7. For any $c \in \mathbb{C}$ there exists a unique structure of quantum vertex algebra on $\mathcal{V}_{c}(\bar{R})$ such that the vacuum vector is $\mathbf{1} \in \mathcal{V}_{c}(\bar{R})$, the vertex operator map is defined by

$$
Y\left(T_{[n]}^{+}(u) \mathbf{1}, z\right)=\mathcal{T}_{[n]}(u \mid z)
$$

the map $D$ is defined by

$$
e^{z D} T_{[n]}^{+}(u) \mathbf{1}=T_{[n]}^{+}(u \mid z) \mathbf{1}
$$

and the map $\mathcal{S}(z)$ is defined by

$$
\begin{gathered}
\mathcal{S}(z)\left(\bar{R}_{n m}^{12}(u|v| z)^{-1} T_{[m]}^{+24}(v) \bar{R}_{n m}^{12}(u|v| z-h c) T_{[n]}^{+13}(u)(\mathbf{1} \otimes \mathbf{1})\right) \\
=T_{[n]}^{+13}(u) \bar{R}_{n m}^{12}(u|v| z+h c)^{-1} T_{[m]}^{+24}(v) \bar{R}_{n m}^{12}(u|v| z)(\mathbf{1} \otimes \mathbf{1})
\end{gathered}
$$

for operators on $\left(\text { End } \mathbb{C}^{N}\right)^{\otimes n} \otimes\left(\text { End } \mathbb{C}^{N}\right)^{\otimes m} \otimes \mathcal{V}_{c}(\bar{R}) \otimes \mathcal{V}_{c}(\bar{R})$.

Proof. Since maps (3.12) and (3.13) coincide with the original maps from [4, Theorem 2.3], we only have to check that (3.11) coincides with the original definition of the vertex operator map in [4], which is given by

$$
T_{[n]}^{+}(u) \mathbf{1} \mapsto T_{[n]}^{+}(u \mid z) T_{[n]}(u \mid z+h c / 2)^{-1} \text { for } n \geqslant 1 .
$$

Due to Poincaré-Birkhoff-Witt theorem for the double Yangian [9, Theorem 2.2], it is sufficient to prove that for any integer $m \geqslant 1$ the actions of (3.11) and (3.14) on 
$\mathcal{T}_{[m]}(v) \mathbf{1} \equiv T_{[m]}^{+}(v) \mathbf{1}$, where $v=\left(v_{1}, \ldots, v_{m}\right)$, coincide. By applying $(3.14)$ on $T_{[m]}^{+}(v) \mathbf{1}$ and using relation (1.21), together with crossing symmetry property (1.8), we obtain

$$
T_{[n]}^{+13}(u \mid z) T_{[n]}^{13}(u \mid z+h c / 2)^{-1} T_{[m]}^{+23}(v) \mathbf{1}=A_{\mathrm{RL}} \cdot\left(T_{[n]}^{+13}(u \mid z) T_{[m]}^{+23}(v) \mathbf{1} \cdot B\right),
$$

where

$$
A=\bar{R}_{n m}^{12}(u|v| z+h c+h N) \quad \text { and } \quad B=\bar{R}_{n m}^{12}(u|v| z)^{-1}
$$

On the other hand, by applying (3.11) on $\mathcal{T}_{[m]}(v) \mathbf{1}$ and using commutation relation (3.9), together with crossing symmetry property (1.8), we get

$$
\mathcal{T}_{[n]}^{13}(u \mid z) \mathcal{T}_{[m]}^{23}(v) \mathbf{1}=A_{\mathrm{RL}}\left(\mathcal{T}_{[n+m]}\left(z+u_{1}, \ldots, z+u_{n}, v_{1}, \ldots, v_{m}\right) \mathbf{1} \cdot B\right) .
$$

Since $\mathbb{C}[[h]]$-module isomorphism (3.3) maps the right hand side of (3.16) to the right hand side of (3.15), definitions in (3.11) and in (3.14) coincide, as required.

3.3. Main result. In the following lemma, we introduce certain map $\mathcal{M}(z)$ which is used in the proof of Theorem 3.9.

Lemma 3.8. For any $c \in \mathbb{C}$ the assignments

$$
T_{[n]}^{+13}(u) T_{[m]}^{+24}(v)(\mathbf{1} \otimes \mathbf{1}) \mapsto \bar{R}_{n m}^{12}(u|v| z)^{-1} T_{[m]}^{+23}(v) \bar{R}_{n m}^{12}(u|v| z-h c) T_{[n]}^{+14}(u)(\mathbf{1} \otimes \mathbf{1})
$$

with $n, m \geqslant 1$ and the variables $u=\left(u_{1}, \ldots, u_{n}\right)$ and $v=\left(v_{1}, \ldots, v_{m}\right)$ define a $\mathbb{C}[[h]]$ module map

$$
\mathcal{M}(z): \mathcal{V}_{c}(\bar{R}) \otimes \mathcal{V}_{c}(\bar{R}) \rightarrow \mathcal{V}_{c}(\bar{R}) \otimes \mathcal{V}_{c}(\bar{R}) \otimes \mathbb{C}((z))
$$

Moreover, the following equalities hold:

$$
\begin{aligned}
& Y(z)\left(\mathcal{M}(-z)\left(T_{[n]}^{+13}(u) T_{[m]}^{+24}(v)(\mathbf{1} \otimes \mathbf{1})\right)\right)=T_{[n]}^{+13}(u) T_{[m]}^{+23}(v \mid z) \mathbf{1} ; \\
& \mathcal{S}(z)\left(P^{\prime}\left(\mathcal{M}(z)\left(T_{[n]}^{+13}(u) T_{[m]}^{+24}(v)(\mathbf{1} \otimes \mathbf{1})\right)\right)\right) \\
& \quad=T_{[n]}^{+13}(u) \bar{R}_{n m}^{12}(u|v| z+h c)^{-1} T_{[m]}^{+24}(v) \bar{R}_{n m}^{12}(u|v| z)(\mathbf{1} \otimes \mathbf{1})
\end{aligned}
$$

for operators on $\left(\text { End } \mathbb{C}^{N}\right)^{\otimes n} \otimes\left(\text { End } \mathbb{C}^{N}\right)^{\otimes m} \otimes \mathcal{V}_{c}(\bar{R}) \otimes \mathcal{V}_{c}(\bar{R})$, where $P^{\prime}: w_{1} \otimes w_{2} \mapsto w_{2} \otimes w_{1}$ denotes the permutation operator on $\mathcal{V}_{c}(\bar{R}) \otimes \mathcal{V}_{c}(\bar{R})$.

Proof. The fact that $\mathbb{C}[[h]]$-module map (3.18) is well-defined can be proved by a simple calculation which relies on Yang-Baxter equation (1.6) and defining relations (1.10) for the dual Yangian. The proof of equalities (3.19) and (3.20) is also straightforward. The former employs unitarity property (1.5) and relations (1.19) and (1.21) while the latter follows directly from (3.13).

The following theorem is the main result of this paper.

Theorem 3.9. Let $W$ be a restricted $\mathrm{A}(\bar{R})$-module of level $c \in \mathbb{C}$. There exists a unique structure of $\mathcal{V}_{c}(\bar{R})$-module on $W$ satisfying

$$
Y_{W}\left(T_{[n]}^{+}(u) \mathbf{1}, z\right)=\mathcal{T}_{[n]}(u \mid z)
$$

for all $n \geqslant 1$. Conversely, let $\left(W, Y_{W}\right)$ be a $\mathcal{V}_{c}(\bar{R})$-module for some $c \in \mathbb{C}$. There exists a unique structure of restricted $\mathrm{A}(\bar{R})$-module of level $c$ on $W$ satisfying

$$
\mathcal{T}(z)=\underset{22}{Y_{W}\left(T^{+}(0) \mathbf{1}, z\right) .}
$$


Moreover, a topologically free $\mathbb{C}[[h]]$-submodule $W_{1}$ of $W$ is a $\mathcal{V}_{c}(\bar{R})$-submodule of $W$ if and only if $W_{1}$ is an $\mathrm{A}(\bar{R})$-submodule of $W$.

Proof. Let $W$ be a restricted $\mathrm{A}(\bar{R})$-module of level $c \in \mathbb{C}$. Equalities (3.21) with $n \geqslant 1$, together with $Y_{W}(\mathbf{1}, z)=1_{W}$, define a $\mathbb{C}[[h]]$-module map $Y_{W}(z): V \otimes W \rightarrow W((z))[[h]]$. Indeed, the fact that $Y_{W}(z)$ is well-defined can be verified by a simple calculation which relies on defining relations (1.10) for the dual Yangian and (3.10).

We now prove that the map $Y_{W}(z)$, as defined by (3.21), satisfies weak associativity (1.32). Let $w$ be an arbitrary element of $W$. Consider the expression

$$
Y_{W}\left(T_{[n]}^{+13}(u) \mathbf{1}, z_{0}+z_{2}\right) Y_{W}\left(T_{[m]}^{+23}(v) \mathbf{1}, z_{2}\right) w
$$

which corresponds to the first summand in (1.32). By (3.21), the given expression equals

$$
\mathcal{T}_{[n]}^{13}\left(u \mid z_{0}+z_{2}\right) \mathcal{T}_{[m]}^{23}\left(v \mid z_{2}\right) w .
$$

By combining (3.9) and crossing symmetry property (1.8) we express (3.24) as

$$
\bar{R}_{n m}^{12}\left(u|v| z_{0}+h c+h N\right) \underset{\mathrm{RL}}{\cdot}\left(\mathcal{T}_{[n+m]}(x) w \cdot \bar{R}_{n m}^{12}\left(u|v| z_{0}\right)^{-1}\right),
$$

where the variables $x=\left(x_{1}, \ldots, x_{n+m}\right)$ are given by

$$
x=\left(z_{0}+z_{2}+u_{1}, \ldots, z_{0}+z_{2}+u_{n}, z_{2}+v_{1}, \ldots, z_{2}+v_{m}\right) .
$$

Next, consider the expression

$$
Y_{W}\left(Y\left(T_{[n]}^{+13}(u) \mathbf{1}, z_{0}\right) T_{[m]}^{+23}(v) \mathbf{1}, z_{2}\right) w,
$$

which corresponds to the second summand in (1.32). By using (3.11) and the identification $T_{[m]}^{+}(v) \mathbf{1} \equiv \mathcal{T}_{[m]}^{+}(v) \mathbf{1}$ we express $(3.26)$ as

$$
Y_{W}\left(\mathcal{T}_{[n]}^{13}\left(u \mid z_{0}\right) \mathcal{T}_{[m]}^{23}(v) \mathbf{1}, z_{2}\right) w .
$$

As before, we employ (3.9) and crossing symmetry property (1.8) to write (3.27) as

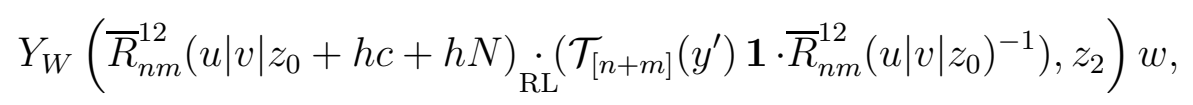

where the variables $y^{\prime}=\left(y_{1}^{\prime}, \ldots, y_{n+m}^{\prime}\right)$ are given by

$$
y^{\prime}=\left(z_{0}+u_{1}, \ldots, z_{0}+u_{n}, v_{1}, \ldots, v_{m}\right) .
$$

Due to (3.21), the expression in (3.28) is equal to

$$
\bar{R}_{n m}^{12}\left(u|v| z_{0}+h c+h N\right)_{\mathrm{RL}}\left(\mathcal{T}_{[n+m]}(y) w \cdot \bar{R}_{n m}^{12}\left(u|v| z_{0}\right)^{-1}\right),
$$

where the variables $y=\left(y_{1}, \ldots, y_{n+m}\right)$ are given by

$$
y=\left(z_{2}+z_{0}+u_{1}, \ldots, z_{2}+z_{0}+u_{n}, z_{2}+v_{1}, \ldots, z_{2}+v_{m}\right) .
$$

Observe that (3.25) and (3.29) are not equal. Indeed, due to our expansion convention from Section 1.3, the former is expanded in nonnegative powers of the variable $z_{2}$ while the latter is expanded in nonnegative powers of the variable $z_{0}$. Fix arbitrary nonnegative integers $k, r_{1}, \ldots, r_{n}, s_{1}, \ldots, s_{m}$. Since $W$ is a restricted $\mathrm{A}(\bar{R})$-module, Proposition 3.5 implies that the coefficient of the monomial $u_{1}^{r_{1}} \ldots u_{n}^{r_{n}} v_{1}^{s_{1}} \ldots v_{m}^{s_{m}}$ in

$$
\mathcal{T}_{[n+m]}\left(z+u_{1}, \ldots, z+u_{n}, v_{1}, \ldots v_{m}\right) w
$$


possesses only finitely many negative powers of the variable $z$ modulo $h^{k}$. Choose an integer $r \geqslant 0$ such that the coefficient of $u_{1}^{r_{1}} \ldots u_{n}^{r_{n}} v_{1}^{s_{1}} \ldots v_{m}^{s_{m}}$ in

$$
z^{r} \mathcal{T}_{[n+m]}\left(z+u_{1}, \ldots, z+u_{n}, v_{1}, \ldots v_{m}\right) w
$$

possesses only nonnegative powers of the variable $z$ modulo $h^{k} .4$ Then, by our discussion, the coefficients of the monomial $u_{1}^{r_{1}} \ldots u_{n}^{r_{n}} v_{1}^{s_{1}} \ldots v_{m}^{s_{m}}$ in

$$
\left(z_{0}+z_{2}\right)^{r} Y_{W}\left(T_{[n]}^{+13}(u) \mathbf{1}, z_{0}+z_{2}\right) Y_{W}\left(T_{[m]}^{+23}(v) \mathbf{1}, z_{2}\right) w
$$

and in

$$
\left(z_{0}+z_{2}\right)^{r} Y_{W}\left(Y\left(T_{[n]}^{+13}(u) \mathbf{1}, z_{0}\right) T_{[m]}^{+23}(v) \mathbf{1}, z_{2}\right) w
$$

coincide modulo $h^{k}$, so we conclude that weak associativity (1.32) holds. Hence, Lemma 1.3 implies that $\left(W, Y_{W}\right)$ is a $\mathcal{V}_{c}(\bar{R})$-module.

Conversely, let $\left(W, Y_{W}\right)$ be a $\mathcal{V}_{c}(\bar{R})$-module. For an arbitrary $w \in W$ let us apply Jacobi identity (1.31) on the last three tensor factors of the expression

$$
\begin{aligned}
A\left(z_{0}\right) & :=\mathcal{M}\left(-z_{0}\right)\left(T_{13}^{+}(u) T_{24}^{+}(v)(\mathbf{1} \otimes \mathbf{1})\right) \otimes w \\
& =\bar{R}\left(-z_{0}+u-v\right)^{-1} T_{23}^{+}(v) \bar{R}\left(-z_{0}+u-v-h c\right) T_{14}^{+}(u)(\mathbf{1} \otimes \mathbf{1}) \otimes w,
\end{aligned}
$$

which belongs to $\left(\text { End } \mathbb{C}^{N}\right)^{\otimes 2} \otimes \mathcal{V}_{c}(\bar{R})^{\otimes 2} \otimes W\left(\left(z_{0}\right)\right)[[u, v, h]]$. By applying the first term in (1.31),

$$
z_{0}^{-1} \delta\left(\frac{z_{1}-z_{2}}{z_{0}}\right) Y_{W}\left(z_{1}\right)\left(1 \otimes Y_{W}\left(z_{2}\right)\right)
$$

on $A\left(z_{0}\right)$, as given in (3.32), we get

$$
\begin{aligned}
& z_{0}^{-1} \delta\left(\frac{z_{1}-z_{2}}{z_{0}}\right) \bar{R}\left(-z_{1}+z_{2}+u-v\right)^{-1} Y_{W}\left(T_{2}^{+}(v) \mathbf{1}, z_{1}\right) \\
& \quad \times \bar{R}\left(-z_{1}+z_{2}+u-v-h c\right) Y_{W}\left(T_{1}^{+}(u) \mathbf{1}, z_{2}\right) w .
\end{aligned}
$$

By applying the second term in (1.31),

$$
-z_{0}^{-1} \delta\left(\frac{z_{2}-z_{1}}{-z_{0}}\right) Y_{W}\left(z_{2}\right)\left(1 \otimes Y_{W}\left(z_{1}\right)\right)\left(\mathcal{S}\left(-z_{0}\right) P^{\prime} \otimes 1\right)
$$

on $A\left(z_{0}\right)$, as given in (3.31), and using (3.20) we get

$$
\begin{aligned}
& -z_{0}^{-1} \delta\left(\frac{z_{2}-z_{1}}{-z_{0}}\right) Y_{W}\left(T_{1}^{+}(u) \mathbf{1}, z_{2}\right) \\
& \quad \times \bar{R}\left(z_{2}-z_{1}+u-v+h c\right)^{-1} Y_{W}\left(T_{2}^{+}(v) \mathbf{1}, z_{1}\right) \bar{R}\left(z_{2}-z_{1}+u-v\right) w .
\end{aligned}
$$

Finally, by applying the third term in (1.31),

$$
z_{2}^{-1} \delta\left(\frac{z_{1}-z_{0}}{z_{2}}\right) Y_{W}\left(z_{2}\right)\left(Y\left(z_{0}\right) \otimes 1\right)
$$

on $A\left(z_{0}\right)$, as given in (3.31), and using (3.19) we get

$$
z_{2}^{-1} \delta\left(\frac{z_{1}-z_{0}}{z_{2}}\right) Y_{W}\left(T_{1}^{+}(u) T_{2}^{+}\left(z_{0}+v\right) \mathbf{1}, z_{2}\right) w .
$$

\footnotetext{
${ }^{4}$ Notice that the integer $r \geqslant 0$ also depends on the choice of $w \in W$.
} 
Since (3.35) does not contain any negative powers of the variable $z_{0}$, the sum of residues of expressions (3.33) and (3.34), with respect to the variable $z_{0}$, equals 0 . Therefore, by taking the residue $\operatorname{Res}_{z_{0}}$ we obtain

$$
\begin{aligned}
& \bar{R}\left(-z_{1}+z_{2}+u-v\right)^{-1} Y_{W}\left(T_{2}^{+}(v) \mathbf{1}, z_{1}\right) \bar{R}\left(-z_{1}+z_{2}+u-v-h c\right) Y_{W}\left(T_{1}^{+}(u) \mathbf{1}, z_{2}\right) w \\
= & Y_{W}\left(T_{1}^{+}(u) \mathbf{1}, z_{2}\right) \bar{R}\left(z_{2}-z_{1}+u-v+h c\right)^{-1} Y_{W}\left(T_{2}^{+}(v) \mathbf{1}, z_{1}\right) \bar{R}\left(z_{2}-z_{1}+u-v\right) w .
\end{aligned}
$$

Both sides of this equality contain only nonnegative powers of the variables $u$ and $v$. In particular, their constant terms, with respect to $u$ and $v$, coincide, i.e. we have

$$
\begin{aligned}
& \bar{R}\left(-z_{1}+z_{2}\right)^{-1} Y_{W}\left(T_{2}^{+}(0) \mathbf{1}, z_{1}\right) \bar{R}\left(-z_{1}+z_{2}-h c\right) Y_{W}\left(T_{1}^{+}(0) \mathbf{1}, z_{2}\right) w \\
= & Y_{W}\left(T_{1}^{+}(0) \mathbf{1}, z_{2}\right) \bar{R}\left(z_{2}-z_{1}+h c\right)^{-1} Y_{W}\left(T_{2}^{+}(0) \mathbf{1}, z_{1}\right) \bar{R}\left(z_{2}-z_{1}\right) w .
\end{aligned}
$$

Since the element $w \in W$ was arbitrary, the following equality holds on $W$ :

$$
\begin{aligned}
& \bar{R}\left(-z_{1}+z_{2}\right)^{-1} Y_{W}\left(T_{2}^{+}(0) \mathbf{1}, z_{1}\right) \bar{R}\left(-z_{1}+z_{2}-h c\right) Y_{W}\left(T_{1}^{+}(0) \mathbf{1}, z_{2}\right) \\
= & Y_{W}\left(T_{1}^{+}(0) \mathbf{1}, z_{2}\right) \bar{R}\left(z_{2}-z_{1}+h c\right)^{-1} Y_{W}\left(T_{2}^{+}(0) \mathbf{1}, z_{1}\right) \bar{R}\left(z_{2}-z_{1}\right) .
\end{aligned}
$$

Observe that (3.36) coincides with commutation relation (2.8) at $C=c$. Therefore, since $Y_{W}\left(T^{+}(0) \mathbf{1}, z\right) w$ belongs to End $\mathbb{C}^{N} \otimes W((z))[[h]]$ for all $w \in W$, we conclude that $(3.22)$ defines a structure of a level $c$ restricted $\mathrm{A}(\bar{R})$-module on $W$.

Finally, let us prove the last assertion of the theorem. Suppose that $W_{1}$ is a $\mathcal{V}_{c}(\bar{R})$ submodule of $W$. Then for any $w_{1} \in W_{1}$ we have

$$
\mathcal{T}(z) w_{1}=Y_{W}\left(T^{+}(0) \mathbf{1}, z\right) w_{1} \in \text { End } \mathbb{C}^{N} \otimes W_{1}((z))[[h]],
$$

so $W_{1}$ is an $\mathrm{A}(\bar{R})$-submodule of $W$. Conversely, suppose that $W_{1}$ is a topologically free $\mathrm{A}(\bar{R})$-submodule of $W$. Clearly, $W_{1}$ is a restricted $\mathrm{A}(\bar{R})$-module (of level $c$ ), so Proposition 3.5 implies that for any $w_{1} \in W_{1}$ we have

$$
\mathcal{T}_{[n]}(v) w_{1} \in\left(\text { End } \mathbb{C}^{N}\right)^{\otimes n} \otimes W_{1}\left(\left(v_{1}, \ldots, v_{n}\right)\right)[[h]] \text { for all } n \geqslant 1
$$

By substituting the variables $v=\left(v_{1}, \ldots, v_{n}\right)$ with $\left(z+u_{1}, \ldots z+u_{n}\right)$, for some variables $u=\left(u_{1}, \ldots, u_{n}\right)$ and $z$, we get, due to the expansion convention from Section 1.3,

$$
Y_{W}\left(T_{[n]}^{+}(u) \mathbf{1}, z\right) w_{1}=\mathcal{T}_{[n]}(u \mid z) w_{1} \in\left(\text { End } \mathbb{C}^{N}\right)^{\otimes n} \otimes W_{1}((z))\left[\left[u_{1}, \ldots, u_{n}, h\right]\right]
$$

for all $n \geqslant 1$. Hence $W_{1}$ is a $\mathcal{V}_{c}(\bar{R})$-submodule of $W$.

\section{ACKNOWLEDGEMENT}

The author would like to thank Alexander Molev for useful discussions. We would also like to thank the anonymous referee for useful suggestions which helped us to improve the manuscript. The research was partially supported by the Croatian Science Foundation under the project 2634 and by the Australian Research Council. 


\section{REFERENCES}

[1] I. V. Cherednik, A new interpretation of Gelfand-Tzetlin bases, Duke Math. J. 54 (1987), 563-577.

[2] J. Ding, Spinor Representations of $U_{q}(\hat{\mathfrak{g l}}(n))$ and Quantum Boson-Fermion Correspondence, Comm. Math. Phys. 200 (1999), 399-420; arXiv:q-alg/9510014.

[3] P. Etingof and D. Kazhdan, Quantization of Lie bialgebras, IV, Selecta Math. (N.S.) 6 (2000), 79-104; arXiv:math/9801043 [math.QA].

[4] P. Etingof, D. Kazhdan, Quantization of Lie bialgebras, V, Selecta Math. (N.S.) 6 (2000), 105-130; arXiv:math/9808121 [math.QA].

[5] L. Frappat, N. Jing, A. Molev and E. Ragoucy, Higher Sugawara operators for the quantum affine algebras of type A, Comm. Math. Phys. 345 (2016), 631-657; arXiv:1505.03667 [math.QA].

[6] I. B. Frenkel and Y.-C. Zhu, Vertex operator algebras associated to representations of affine and Virasoro algebras, Duke Math. J. 66 (1992), 123-168.

[7] D. Gurevich, P. Saponov, Centers in Generalized Reflection Equation algebras, arXiv:1712.06154 [math.QA].

[8] K. Iohara, Bosonic representations of Yangian double $D Y_{\hbar}(\mathfrak{g})$ with $\mathfrak{g}=\mathfrak{g l}_{N}, \mathfrak{s l}{ }_{N}$, J. Phys. A 29 (1996), 4593-4621; arXiv:q-alg/9603033.

[9] N. Jing, S. Kožić, A. Molev, F. Yang, Center of the quantum affine vertex algebra in type A, J. Algebra 496 (2018), 138-186; arXiv:1603.00237 [math.QA].

[10] D. Jordan, N. White, The center of the reflection equation algebra via quantum minors, arXiv:1709.09149 [math.QA].

[11] A. Jucys, On the Young operators of the symmetric group, Lietuvos Fizikos Rinkinys 6 (1966), 163-180.

[12] C. Kassel, Quantum Groups, Graduate texts in mathematics; vol. 155, Springer-Verlag, 1995.

[13] S. M. Khoroshkin, Central Extension of the Yangian Double, arXiv:q-alg/9602031.

[14] S. Kožić, Quasi modules for the quantum affine vertex algebra in type A, Comm. Math. Phys. 365 (2019), 1049-1078; arXiv:1707.09542 [math.QA].

[15] P. P. Kulish and E. K. Sklyanin, Algebraic structures related to reflection equations, J. Phys. A 25 (1992), 5963-5975; arXiv:hep-th/9209054.

[16] V. B. Kuznetsov, M. F. Jørgensen, P. L. Christiansen, New boundary conditions for integrable lattices, J. Phys. A 28 (1995), 4639-4654; arXiv:hep-th/9503168.

[17] J. Lepowsky, H.-S. Li, Introduction to Vertex Operator Algebras and Their Representations, Progress in Math., Vol. 227, Birkhauser, Boston, 2004.

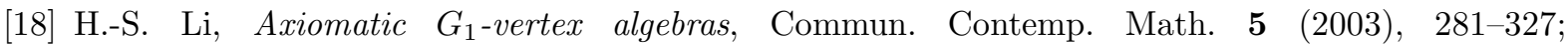
arXiv:math/0204308 [math.QA].

[19] H.-S. Li, Nonlocal vertex algebras generated by formal vertex operators, Selecta Math. (New Series) 11 (2005), 349-397; arXiv:math/0502244 [math.QA].

[20] H.-S. Li, Constructing quantum vertex algebras, Int. J. Math. 17 (2006), 441-476; arXiv:math/0505293 [math.QA].

[21] H.-S. Li, ћ-adic quantum vertex algebras and their modules, Comm. Math. Phys. 296 (2010), 475523; arXiv:0812.3156 [math.QA].

[22] B.-H. Lian, On the classification of simple vertex operator algebras, Comm. Math. Phys. 163 (1994), 307-357.

[23] M. Mintchev, E. Ragoucy and P. Sorba, Spontaneous symmetry breaking in the gl(N) - NLS hierarchy on the half line, J. Phys. A 34 (2001) 8345-8364; arXiv:hep-th/0104079.

[24] A. Molev, Yangians and classical Lie algebras, Mathematical Surveys and Monographs, 143. American Mathematical Society, Providence, RI, 2007.

[25] A. I. Molev, E. Ragoucy, Representations of reflection algebras, Rev. Math. Phys. 14 (2002), 317342; arXiv:math/0107213 [math.QA]. 
[26] A. Okounkov, Quantum immanants and higher Capelli identities, Transform. Groups 1 (1996), 99126; arXiv:q-alg/9602028.

[27] N. Yu. Reshetikhin, M. A. Semenov-Tian-Shansky, Central extensions of quantum current groups, Lett. Math. Phys., 19 (1990), 133-142.

[28] E. K. Sklyanin, Boundary conditions for integrable quantum systems, J. Phys. A 21 (1988), 23752389.

[29] D. V. Talalaev, The quantum Gaudin system, Funct. Anal. Appl. 40 (2006), 73-77.

Department of Mathematics, Faculty of Science, University of Zagreb, 10000 Zagreb, Croatia and School of Mathematics and Statistics F07, University of Sydney, NSW 2006, Australia

E-mail address: kslaven@math.hr 